K. W. MORRIS (Adelaide)

D. Szynal (Lublin)

\title{
GOODNESS-OF-FIT TESTS BASED ON CHARACTERIZATIONS IN TERMS OF MOMENTS OF ORDER STATISTICS
}

Abstract. Using characterization conditions of continuous distributions in terms of moments of order statistics given in [12], [23], [6] and [7] we present new goodness-of-fit techniques.

1. Introduction and preliminaries. Let $\left(X_{1}, \ldots, X_{n}\right)$ be a sample from a continuous distribution $F(x)=P[X \leq x], x \in \mathbb{R}$. There are many methods for constructing goodness-of-fit tests (cf. [4], [20], [21]), including methods using characterizations of distributions (cf. [2], [3], [8], [13]). We give tests of fit based on characterizations of continuous distributions via moments of order statistics (cf. [6], [7], [12], [23]). The tests presented are asymptotic and they treat cases where the parameters of the distribution must be estimated from the data. The proposed approach avoids the difficulty of determining for instance the number of classes, the number of summands, or the window size, associated with some recommended tests ( $\chi^{2}$-tests, data-driven Neyman's tests, tests based on entropy, etc.) (cf. [4], $[9],[5])$. We present a family of tests depending on a parameter $r>0$, the order of a moment of a certain variable. The power of these tests depends on $r$. In the case $r=1$ we obtain tests discussed in previous papers [14]-[17].

2. Characterization conditions. Let $\left(X_{1}, \ldots, X_{n}\right)$ be a sample from a continuous distribution $F(x)=P[X \leq x], x \in \mathbb{R}$, and let $X_{k: n}$ denote the $k$ th smallest order statistic of the sample. We use the following char-

2000 Mathematics Subject Classification: Primary 62E10, 62F03.

Key words and phrases: order statistics, moments, characterizations, uniform, power, exponential, Weibull, Pareto, logistic, Cauchy, normal, extreme-value distributions, maximum likehood estimators, goodness-of-fit tests. 
acterizations of continuous distributions via moments of functions of order statistics.

Theorem 1 (cf. [23], [6], [7]). Let $n, k, l$ be given integers such that $n \geq k \geq l \geq 1$. Assume that $G$ is a nondecreasing and right-continuous function from $\mathbb{R}$ to $\mathbb{R}$. Then $F(x)=G(x)$ on $I(F)$ (the minimal interval containing the support of $F$ ) and $F$ is continuous on $\mathbb{R}$ iff

$$
\begin{aligned}
\frac{(k-l) !}{(n-l+1) !} E G^{2 l}\left(X_{k+1-l: n+1-l}\right)-\frac{2 k !}{(n+1) !} & E G^{l}\left(X_{k+1: n+1}\right) \\
& +\frac{(k+l) !}{(n+l+1) !}=0 .
\end{aligned}
$$

TheOREM 2 (cf. [12]). Under the assumptions of Theorem 1, $F(x)=$ $G(x)$ on $I(F)$ and $F$ is continuous on $\mathbb{R}$ iff

$$
\begin{aligned}
& E G^{l}\left(X_{k+1: n+1}\right)=\frac{(k+l) !(n+1) !}{k !(n+l+1) !} \\
& E G^{2 l}\left(X_{k+1-l: n+1-l}\right)=\frac{(k+l) !(n-l+1) !}{(k-l) !(n+l+1) !} .
\end{aligned}
$$

Note that Theorem 2 is a consequence of Theorem 1, since (2.1) implies $F=G$ implies (2.2) implies (2.1).

Corollary 1. $X \sim F$ and $F$ is continuous iff

$$
E F\left(X_{2: 2}\right)-E F^{2}(X)=\frac{1}{3}
$$

or iff

$$
E F\left(X_{2: 2}\right)=\frac{2}{3}, \quad E F^{2}(X)=\frac{1}{3} .
$$

We also need the following generalization of Theorems 2 and 1.

Theorem 3 (cf. [12], [7]). Let $\left(X_{1}, \ldots, X_{n}\right)$ be a sample from a distribution $F$ and assume that $G$ is a nondecreasing and right-continuous function from $\mathbb{R}$ to $[0, \infty)$. Let $n \geq k \geq 2$ be given positive integers and $r$, $p, q$ given positive real numbers such that $p>1, q>1,1 / p+1 / q=1$, and $s=p(q(k-1)-p r) /(p+q)$ is a nonnegative integer. Then $F(x)=G(x)$ on $I(F)$ and $F$ is continuous on $\mathbb{R}$ iff

$$
\begin{aligned}
& E G^{r}\left(X_{k: n}\right)=\frac{B(k+r, n-k+1)}{B(k, n-k+1)}, \\
& E G^{p r}\left(X_{s+1: n-k+s+1}\right)=\frac{B(k+r, n-k+1)}{B(s+1, n-k+1)},
\end{aligned}
$$


where

$$
B(a, b)=\int_{0}^{1} t^{a-1}(1-t)^{b-1} d t, \quad a>0, b>0,
$$

is the Beta function, or iff

$$
\begin{array}{r}
\frac{s !}{(n-k+s+1) !} E G^{p r}\left(X_{s+1: n-k+s+1}\right)-\frac{2(k-1) !}{n !} E G^{r}\left(X_{k: n}\right) \\
+\frac{\Gamma(k+r)}{\Gamma(n+r+1)}=0 .
\end{array}
$$

Corollary 2. Taking $k=n=2, s=0$ in (2.5) and (2.5'), we have, for given $r>0, p=(r+1) / r$ and $X \sim F$ iff

$$
E F^{r}\left(X_{2: 2}\right)=\frac{2}{2+r}, \quad E F^{r+1}(X)=\frac{1}{2+r}
$$

or iff

$$
E F^{r}\left(X_{2: 2}\right)-E F^{1+r}(X)=\frac{1}{2+r}
$$

The following statement will also be used.

REMARK. If $X \sim F$ then

$$
\frac{1}{r} E F^{r}\left(X_{2: 2}\right)-\frac{2}{1+r} E F^{1+r}(X)=\frac{2}{r(1+r)(2+r)} .
$$

\section{Goodness-of-fit tests based on Corollary 2}

(A) Parameters of $F$ are specified. Let $\left(X_{1}, \ldots, X_{2 n}\right)$ be a sample from $F$, where $F$ is continuous and strictly increasing. For $r>0$ define

$$
\begin{aligned}
Y_{j}^{(r)} & =F^{1+r}\left(X_{2 j-1}\right)+F^{1+r}\left(X_{2 j}\right), \\
Z_{j}^{(r)} & =F^{r}\left(\max \left(X_{2 j-1}, X_{2 j}\right)\right), \quad j=1, \ldots, n .
\end{aligned}
$$

Then $Y_{1}^{(r)}, \ldots, Y_{n}^{(r)}$ are i.i.d. and $Z_{1}^{(r)}, \ldots, Z_{n}^{(r)}$ are i.i.d., and by (2.6),

$$
E Y_{j}^{(r)}=\frac{2}{2+r}, \quad E Z_{j}^{(r)}=\frac{2}{2+r}, \quad j=1, \ldots, n .
$$

For

$$
Y:=Y_{1}^{(r)}=F^{1+r}\left(X_{1}\right)+F^{1+r}\left(X_{2}\right), \quad Z:=Z_{1}^{(r)}=F^{r}\left(\max \left(X_{1}, X_{2}\right)\right),
$$

we have the following result. 
Lemma 1 (cf. [15], [16]). The density function of $(Y, Z)$ is given by

$$
f_{Y, Z}(y, z)=\left\{\begin{array}{l}
\frac{2}{r(1+r) z^{1-1 / r}\left(y-z^{1+1 / r}\right)^{1-1 /(1+r)}}, \\
z^{1+1 / r} \leq y \leq 2 z^{1+1 / r}, 0 \leq z \leq 1, \\
\text { otherwise, }
\end{array}\right.
$$

and

$$
\begin{aligned}
& E Y=\frac{2}{2+r}, \quad \operatorname{Var} Y=\frac{2(1+r)^{2}}{(2+r)^{2}(3+2 r)}, \quad E Z=\frac{2}{2+r}, \\
& \operatorname{Var} Z=\frac{r^{2}}{(2+r)^{2}(1+r)}, \quad \operatorname{Cov}(Y, Z)=\frac{2 r(1+r)}{(2+r)^{2}(3+2 r)} .
\end{aligned}
$$

Now we define

$$
\boldsymbol{W}_{j}^{(r)}=\left(\begin{array}{c}
Y_{j}^{(r)} \\
Z_{j}^{(r)}
\end{array}\right), \quad j=1, \ldots, n
$$

We see that

$$
\begin{gathered}
\boldsymbol{\mu}_{r}=E \boldsymbol{W}_{1}^{(r)}=\left(\begin{array}{c}
\frac{2}{2+r} \\
\frac{2}{2+r}
\end{array}\right)=\frac{2}{2+r}\left(\begin{array}{c}
1 \\
1
\end{array}\right), \\
\Sigma_{r}:=\operatorname{Var}\left(\boldsymbol{W}_{1}^{(r)}\right)=\left(\begin{array}{cc}
\frac{2(1+r)^{2}}{(2+r)^{2}(3+2 r)} & \frac{2 r(1+r)}{(2+r)^{2}(3+2 r)} \\
\frac{2 r(1+r)}{(2+r)^{2}(3+2 r)} & \frac{r^{2}}{(2+r)^{2}(1+r)}
\end{array}\right), \\
\Sigma_{r}^{-1}=\frac{(2+r)^{2}(3+2 r)}{2 r^{2}(1+r)^{2}}\left(\begin{array}{cc}
r^{2}(3+2 r) & -2 r(1+r)^{2} \\
-2 r(1+r)^{2} & 2(1+r)^{3}
\end{array}\right) .
\end{gathered}
$$

Write

$$
\overline{\boldsymbol{W}_{n}^{(r)}}=\frac{1}{n} \sum_{j=1}^{n} \boldsymbol{W}_{j}^{(r)} .
$$

The CLT says that

$$
\sqrt{n}\left(\overline{\boldsymbol{W}_{n}^{(r)}}-\boldsymbol{\mu}_{r}\right) \stackrel{D}{\rightarrow} \boldsymbol{V} \sim \mathrm{N}\left(\mathbf{0}, \Sigma_{r}\right)
$$

whence

$$
D_{n r}^{(3)}:=n\left(\overline{\left(\boldsymbol{W}_{n}^{(r)}\right.}-\boldsymbol{\mu}_{r}\right)^{\prime} \Sigma_{r}^{-1}\left(\overline{\boldsymbol{W}_{n}^{(r)}}-\boldsymbol{\mu}_{r}\right) \stackrel{D}{\rightarrow} \boldsymbol{V}^{\prime} \Sigma_{r}^{-1} \boldsymbol{V} \sim \chi^{2}(2) .
$$

Simple algebraic evaluations allow us to write $D_{n r}^{(3)}$ in the form

$$
\begin{aligned}
D_{n r}^{(3)}= & \frac{(2+r)^{2}(3+2 r) n}{2(1+r)^{2}}\left(\overline{Y_{n}^{(r)}}-\frac{2}{2+r}\right)^{2} \\
& +(1+r)(2+r)^{2}(3+2 r) n\left(\frac{1}{r} \overline{Z_{n}^{(r)}}-\frac{1}{1+r} \overline{Y_{n}^{(r)}}-\frac{2}{r(1+r)(2+r)}\right)^{2} .
\end{aligned}
$$


Letting

$$
X_{j}^{*}=\max \left(X_{2 j-1}, X_{2 j}\right), \quad j=1, \ldots, n,
$$

leads to

$$
\begin{aligned}
D_{n r}^{(3)}= & 2 \frac{(2+r)^{2}(3+2 r)}{(1+r)^{2}} n\left(\overline{F^{1+r}\left(X_{2 n}\right)}-\frac{1}{2+r}\right)^{2} \\
& +(1+r)(2+r)^{2}(3+2 r) n \\
& \times\left(\frac{1}{r} \overline{F^{r}\left(X_{n}^{*}\right)}-\frac{2}{1+r} \overline{F^{1+r}\left(X_{2 n}\right)}-\frac{2}{r(1+r)(2+r)}\right)^{2}
\end{aligned}
$$

where

$$
\overline{F^{1+r}\left(X_{2 n}\right)}=\frac{1}{2 n} \sum_{j=1}^{2 n} F^{1+r}\left(X_{j}\right), \quad \overline{F^{r}\left(X_{n}^{*}\right)}=\frac{1}{n} \sum_{j=1}^{n} F^{r}\left(X_{j}^{*}\right) .
$$

Set

$$
\begin{aligned}
& A_{r}^{(0)}=\frac{(2+r)^{2}(3+2 r)}{(1+r)^{2}}, \quad A_{r}^{(1)}=(1+r)(2+r)^{2}(3+2 r), \\
& A_{r}^{(2)}=\frac{1}{r^{3}+r^{2}-r+1} A_{r}^{(1)}
\end{aligned}
$$

and write

$$
\begin{aligned}
(3.5) \quad D_{n r}^{(0)}:= & A_{r}^{(0)} 2 n\left(\overline{F^{1+r}\left(X_{2 n}\right)}-\frac{1}{2+r}\right)^{2}, \\
(3.6) \quad D_{n r}^{(1)}:= & A_{r}^{(1)} n \\
& \times\left(\frac{1}{r} \overline{F^{r}\left(X_{n}^{*}\right)}-\frac{2}{1+r} \overline{F^{1+r}\left(X_{2 n}\right)}-\frac{2}{r(1+r)(2+r)}\right)^{2}, \\
\left(3.6^{\prime}\right) \quad D_{n r}^{(2)}:= & A_{r}^{(2)} 2 n\left(\overline{F^{r}\left(X_{n}^{*}\right)}-\overline{F^{1+r}\left(X_{2 n}\right)}-\frac{1}{2+r}\right)^{2} .
\end{aligned}
$$

Now we note that (2.7) has the form

$$
\frac{1}{r} E F^{r}\left(X_{2: 2}\right)-\frac{1}{1+r}\left(E F^{1+r}\left(X_{1}\right)+E F^{1+r}\left(X_{2}\right)\right)=\frac{2}{r(1+r)(2+r)} .
$$

Letting

$$
R_{j}^{(r)}=\frac{1}{r} Z_{j}^{(r)}-\frac{1}{1+r} Y_{j}^{(r)}, \quad j=1, \ldots, n,
$$

we see that

$$
E R_{j}^{(r)}=\frac{2}{r(1+r)(2+r)}
$$

and

$$
\operatorname{Var} R_{j}^{(r)}=\frac{1}{(1+r)(2+r)^{2}(3+2 r)} .
$$


Put

$$
\overline{R_{n}^{(r)}}=\frac{1}{n} \sum_{j=1}^{n} R_{j}^{(r)}
$$

Then by the CLT

$$
\begin{aligned}
(2+r) & \sqrt{(1+r)(3+2 r) n}\left(\overline{R_{n}^{(r)}}-\frac{2}{r(1+r)(2+r)}\right) \\
= & (2+r) \sqrt{(1+r)(3+2 r) n}\left(\frac{1}{r} \overline{Z_{n}^{(r)}}-\frac{1}{1+r} \overline{Y_{n}^{(r)}}-\frac{2}{r(1+r)(2+r)}\right) \\
& \stackrel{D}{\rightarrow} V \sim \mathrm{N}(0,1),
\end{aligned}
$$

which implies that

$$
D_{n r}^{(1)} \stackrel{D}{\rightarrow} \chi^{2}(1)
$$

Similarly we prove that

$$
D_{n r}^{(2)} \stackrel{D}{\rightarrow} \chi^{2}(1)
$$

The following statements are consequences of $(3.1)-\left(3.7^{\prime}\right)$.

Proposition 1. Let $\left(X_{1}, \ldots, X_{2 n}\right)$ be a sample from an absolutely continuous distribution function $F$. Then

$$
\begin{aligned}
D_{n r}^{(0)}= & A_{r}^{(0)} \frac{1}{2 n}\left(\sum_{j=1}^{2 n} F^{1+r}\left(X_{j}\right)-\frac{2 n}{2+r}\right)^{2} \stackrel{D}{\rightarrow} \chi^{2}(1) \\
D_{n r}^{(1)}= & A_{r}^{(1)} \frac{1}{n}\left(\frac{1}{r} \sum_{j=1}^{n} F^{r}\left(X_{j}^{*}\right)-\frac{1}{1+r} \sum_{j=1}^{2 n} F^{1+r}\left(X_{j}\right)\right. \\
& \left.-\frac{2 n}{r(1+r)(2+r)}\right)^{2} \stackrel{D}{\rightarrow} \chi^{2}(1)
\end{aligned}
$$

$$
\begin{aligned}
& D_{n r}^{(2)}=A_{r}^{(2)} \frac{2}{n}\left(\sum_{j=1}^{n} F^{r}\left(X_{j}^{*}\right)-\frac{1}{2} \sum_{j=1}^{2 n} F^{1+r}\left(X_{j}\right)-\frac{n}{2+r}\right)^{2} \\
& \quad \stackrel{D}{\rightarrow} \chi^{2}(1),
\end{aligned}
$$

(3.10) $\quad D_{n r}^{(3)}=D_{n r}^{(0)}+D_{n r}^{(1)} \stackrel{D}{\rightarrow} \chi^{2}(2)$,

where $A_{r}^{(0)}, A_{r}^{(1)}, A_{r}^{(2)}$ are in (3.4).

Corollary 3. If $r=1$ then

$$
D_{n 1}^{(0)}=\frac{45}{4} \cdot \frac{1}{2 n}\left(\sum_{j=1}^{2 n} F^{2}\left(X_{j}\right)-\frac{2 n}{3}\right)^{2} \stackrel{D}{\rightarrow} \chi^{2}(1),
$$




$$
\begin{aligned}
& D_{n 1}^{(1)}=D_{n 1}^{(2)}=90 \frac{1}{n}\left(\sum_{j=1}^{n} F\left(X_{j}^{*}\right)-\frac{1}{2} \sum_{j=1}^{2 n} F^{2}\left(X_{j}\right)-\frac{n}{3}\right)^{2} \stackrel{D}{\rightarrow} \chi^{2}(1), \\
& D_{n 1}^{(3)}=D_{n 1}^{(0)}+D_{n 1}^{(1)} \stackrel{D}{\rightarrow} \chi^{2}(2) .
\end{aligned}
$$

We list the following special cases of distribution functions (cf. [1], [11]) for which Proposition 1 ((3.8)-(3.10)) applies.

(i) $X \sim \mathrm{U}(\alpha, \beta)$ (uniform distribution),

$$
F(x)=(x-\alpha) /(\beta-\alpha), \quad \alpha<x<\beta ; \alpha, \beta \in \mathbb{R} .
$$

Remark. For $X \sim \mathrm{U}(0,1), F(x)=x$.

(ii) $X \sim \operatorname{Pow}(\alpha)$ (power distribution),

$$
F(x)=1-(1-x / \alpha)^{\alpha}, \quad 0 \leq x \leq \alpha ; 0<\alpha \leq 1 .
$$

(iii) $X \sim \operatorname{Exp}(\alpha)$ (exponential distribution),

$$
F(x)=1-\exp (-\alpha x), \quad x>0 ; \alpha>0 .
$$

(iv) $X \sim \mathrm{W}(\alpha, \beta)$ (Weibull distribution),

$$
F(x)=1-\exp \left(-\alpha x^{\beta}\right), \quad x>0 ; \alpha>0, \beta>0 .
$$

(v) $X \sim \operatorname{Par}_{\mathrm{S}}(\alpha, \sigma)$ (single-parameter Pareto distribution),

$$
F(x)=1-(\sigma / x)^{\alpha}, \quad x>\sigma ; \alpha>0, \sigma>0 .
$$

Remark. For $X \sim \operatorname{Par}_{\mathrm{S}}(\alpha, 1)$,

$$
F(x)=1-(1 / x)^{\alpha}, \quad x>1 ; \alpha>0 .
$$

(vi) $X \sim \operatorname{Par}_{\mathrm{T}}(\alpha, \theta)$ (two-parameter Pareto distribution),

$$
F(x)=1-\left(\theta /(x+\theta)^{\alpha}\right), \quad x>0 ; \alpha>0, \theta>0 .
$$

(vii) $X \sim \log (\alpha, \beta)$ (logistic distribution),

$$
F(x)=[1+\exp (-(x-\alpha) / \beta)]^{-1}, \quad-\infty<x<\infty ; \alpha \in \mathbb{R}, \beta>0 .
$$

(viii) $X \sim \mathrm{C}(\alpha, \beta)$ (Cauchy distribution),

$$
F(x)=\frac{1}{2}+\frac{1}{\pi} \arctan \frac{x-\alpha}{\beta}, \quad x \in \mathbb{R} ; \alpha \in \mathbb{R}, \beta>0 .
$$

(ix) $X \sim \mathrm{N}(\mu, \sigma)$ (normal distribution),

$$
F(x)=\frac{1}{\sigma \sqrt{2 \pi}} \int_{-\infty}^{x} e^{-\frac{(t-\mu)^{2}}{2 \sigma^{2}}} d t, \quad x \in \mathbb{R} ; \mu \in \mathbb{R}, \sigma>0 .
$$

(x) $X \sim \mathrm{EV}(\alpha, \beta)$ (extreme-value distribution),

$$
F(x)=\exp \left(-\exp \left(-\frac{x-\alpha}{\beta}\right)\right), \quad x \in \mathbb{R} ; \alpha \in \mathbb{R}, \beta>0 .
$$


(B) Unknown parameters. Now we discuss some tests when parameters are replaced by estimators.

Proposition 2. Goodness-of-fit tests for $F \in \mathrm{U}(\alpha, \beta)$ are given by

$$
\begin{aligned}
\widehat{D}_{n r}^{(0)}= & A_{r}^{(0)} \frac{1}{2 n}\left(\frac{1}{\left(\widehat{\beta}_{n}-\widehat{\alpha}_{n}\right)^{1+r}} \sum_{j=1}^{2 n}\left(X_{j}-\widehat{\alpha}_{n}\right)^{1+r}-\frac{2 n}{2+r}\right)^{2} \stackrel{D}{\rightarrow} \chi^{2}(1), \\
\widehat{D}_{n r}^{(1)}= & A_{r}^{(1)} \frac{1}{n}\left(\frac{1}{r} \cdot \frac{1}{\left(\widehat{\beta}_{n}-\widehat{\alpha}_{n}\right)^{r}} \sum_{j=1}^{n}\left(X_{j}^{*}-\widehat{\alpha}_{n}\right)^{r}\right. \\
& \left.-\frac{1}{1+r} \cdot \frac{1}{\left(\widehat{\beta}_{n}-\widehat{\alpha}_{n}\right)^{1+r}} \sum_{j=1}^{2 n}\left(X_{j}-\widehat{\alpha}_{n}\right)^{1+r}-\frac{2 n}{r(1+r)(2+r)}\right)^{2} \\
\stackrel{D}{\rightarrow} & \chi^{2}(1), \\
\widehat{D}_{n r}^{(2)}= & A_{r}^{(2)} \frac{2}{n}\left(\frac{1}{\left(\widehat{\beta}_{n}-\widehat{\alpha}_{n}\right)^{r}} \sum_{j=1}^{n}\left(X_{j}^{*}-\widehat{\alpha}_{n}\right)^{r}\right. \\
& \left.-\frac{1}{2\left(\widehat{\beta}_{n}-\widehat{\alpha}_{n}\right)^{1+r}} \sum_{j=1}^{2 n}\left(X_{j}-\widehat{\alpha}_{n}\right)^{1+r}-\frac{n}{2+r}\right)^{2} \stackrel{D}{\rightarrow} \chi^{2}(1), \\
\widehat{D}_{n r}^{(3)}= & \widehat{D}_{n r}^{(0)}+\widehat{D}_{n r}^{(1)} \stackrel{D}{\rightarrow} \chi^{2}(2),
\end{aligned}
$$

where $A_{r}^{(0)}, A_{r}^{(1)}, A_{r}^{(2)}$ are in (3.4), and $\widehat{\alpha}_{n}=\min \left(X_{1}, \ldots, X_{2 n}\right), \widehat{\beta}_{n}=$ $\max \left(X_{1}, \ldots, X_{2 n}\right)$.

Corollary 4. Goodness-of-fit tests for $F \in \mathrm{U}(0, \beta)$ are given by

$$
\begin{aligned}
\widehat{D}_{n r}^{(0)}= & A_{r}^{(0)} \frac{1}{2 n}\left(\frac{1}{\widehat{\beta}_{n}^{1+r}} \sum_{j=1}^{2 n} X_{j}^{1+r}-\frac{2 n}{2+r}\right)^{2} \stackrel{D}{\rightarrow} \chi^{2}(1), \\
\widehat{D}_{n r}^{(1)}= & A_{r}^{(1)} \frac{1}{n}\left(\frac{1}{r \widehat{\beta}_{n}^{r}} \sum_{j=1}^{n}\left(X_{j}^{*}\right)^{r}-\frac{1}{(1+r) \widehat{\beta}_{n}^{1+r}} \sum_{j=1}^{2 n} X_{j}^{1+r}\right. \\
& \left.-\frac{2 n}{r(1+r)(2+r)}\right)^{2} \stackrel{D}{\rightarrow} \chi^{2}(1), \\
\widehat{D}_{n r}^{(2)}= & A_{r}^{(2)} \frac{2}{n}\left(\frac{1}{\widehat{\beta}_{n}^{r}} \sum_{j=1}^{n}\left(X_{j}^{*}\right)^{r}-\frac{1}{2 \widehat{\beta}_{n}^{1+r}} \sum_{j=1}^{2 n} X_{j}^{1+r}-\frac{n}{2+r}\right)^{2} \stackrel{D}{\rightarrow} \chi^{2}(1), \\
\widehat{D}_{n r}^{(3)}= & \widehat{D}_{n r}^{(0)}+\widehat{D}_{n r}^{(1)} \stackrel{D}{\rightarrow} \chi^{2}(2),
\end{aligned}
$$

where $A_{r}^{(0)}, A_{r}^{(1)}, A_{r}^{(2)}$ are in (3.4) and $\widehat{\beta}_{n}=\max \left(X_{1}, \ldots, X_{2 n}\right)$.

The proof of Proposition 2 for $r=1$ was given in [14] and [15]. The case $r>0$ can be established similarly (cf. [16]). Moreover, theoretical con- 
siderations concerning the variance of $\max \left(X_{1}, \ldots, X_{2 n}\right)$ and evidence from simulations lead us to conjecture the following statement.

Proposition 3. Goodness-of-fit tests for $F \in \operatorname{Pow}(\alpha)$ are given by

$$
\begin{aligned}
\widehat{D}_{n r}^{(0)}= & A_{r}^{(0)} \frac{1}{2 n}\left(\sum_{j=1}^{2 n}\left(1-\left(1-\frac{X_{j}}{\widehat{\alpha}_{n}}\right)^{\widehat{\alpha}_{n}}\right)^{1+r}-\frac{2 n}{2+r}\right)^{2} \stackrel{D}{\rightarrow} \chi^{2}(1), \\
\widehat{D}_{n r}^{(1)}= & A_{r}^{(1)} \frac{1}{n}\left(\frac{1}{r} \sum_{j=1}^{n}\left(1-\left(1-\frac{X_{j}^{*}}{\widehat{\alpha}_{n}}\right)^{\widehat{\alpha}_{n}}\right)^{r}\right. \\
& \left.-\frac{1}{1+r} \sum_{j=1}^{2 n}\left(1-\left(1-\frac{X_{j}}{\widehat{\alpha}_{n}}\right)^{\widehat{\alpha}_{n}}\right)^{1+r}-\frac{2 n}{r(1+r)(2+r)}\right)^{2} \stackrel{D}{\rightarrow} \chi^{2}(1), \\
\widehat{D}_{n r}^{(2)}= & A_{r}^{(2)} \frac{2}{n}\left(\sum_{j=1}^{n}\left(1-\left(1-\frac{X_{j}^{*}}{\widehat{\alpha}_{n}}\right)^{\widehat{\alpha}_{n}}\right)^{r}\right. \\
& \left.-\frac{1}{2} \sum_{j=1}^{2 n}\left(1-\left(1-\frac{X_{j}}{\widehat{\alpha}_{n}}\right)^{\widehat{\alpha}_{n}}\right)^{1+r}-\frac{n}{2+r}\right)^{2} \stackrel{D}{\rightarrow} \chi^{2}(1), \\
\widehat{D}_{n r}^{(3)}= & \widehat{D}_{n r}^{(0)}+\widehat{D}_{n r}^{(1)} \stackrel{D}{\rightarrow} \chi^{2}(2),
\end{aligned}
$$

where $A_{r}^{(0)}, A_{r}^{(1)}, A_{r}^{(2)}$ are in $(3.4)$, and $\widehat{\alpha}_{n}=\max \left(X_{1}, \ldots, X_{2 n}\right)$.

In what follows we use a theorem on the asymptotic effect of substituting estimators for parameters in tests proposed in (A).

Theorem 4 (cf. [18], [19]). Let $\widehat{\boldsymbol{T}}_{n}=\boldsymbol{T}_{n}\left(X_{1}, \ldots, X_{n} ; \widehat{\boldsymbol{\lambda}}_{n}\right)$, where $\widehat{\boldsymbol{\lambda}}_{n}=$ $\widehat{\boldsymbol{\lambda}}_{n}\left(X_{1}, \ldots, X_{n}\right)$ is an estimator of a parameter $\boldsymbol{\lambda}$ of the distribution of $X$, and let $\boldsymbol{T}_{n}=\boldsymbol{T}_{n}\left(X_{1}, \ldots, X_{n} ; \boldsymbol{\lambda}\right)$ (here $\boldsymbol{T}_{n}, \boldsymbol{\lambda}$ and $\widehat{\boldsymbol{\lambda}}_{n}$ may be vectors). Suppose that:

(i) For each $\boldsymbol{\lambda}$,

$$
\sqrt{n}\left(\begin{array}{c}
\boldsymbol{T}_{n} \\
\widehat{\boldsymbol{\lambda}}_{n}-\boldsymbol{\lambda}
\end{array}\right) \stackrel{D}{\rightarrow} \boldsymbol{T} \sim \mathrm{N}(\mathbf{0}, \boldsymbol{V})
$$

where

$$
\boldsymbol{V}=\left(\begin{array}{ll}
\boldsymbol{V}_{11} & \boldsymbol{V}_{12} \\
\boldsymbol{V}_{21} & \boldsymbol{V}_{22}
\end{array}\right)
$$

and $\boldsymbol{V}_{22}$ is nonsingular.

(ii) There is a matrix $\boldsymbol{B}$, possibly depending continuously on $\boldsymbol{\lambda}$, such that

$$
\sqrt{n} \widehat{\boldsymbol{T}}_{n}=\sqrt{n} \boldsymbol{T}_{n}+\boldsymbol{B} \sqrt{n}\left(\widehat{\boldsymbol{\lambda}}_{n}-\boldsymbol{\lambda}\right)+o_{p}(1) .
$$


(iii) $\widehat{\boldsymbol{\lambda}}_{n}$ is asymptotically efficient.

Then

$$
\sqrt{n} \widehat{\boldsymbol{T}}_{n} \stackrel{D}{\rightarrow} \boldsymbol{T}^{*} \sim \mathrm{N}\left(\mathbf{0}, \boldsymbol{V}_{11}-\boldsymbol{B} \boldsymbol{V}_{22} \boldsymbol{B}^{\prime}\right) .
$$

Note that (ii) is satisfied when $\boldsymbol{T}_{n}$ is differentiable in $\boldsymbol{\lambda}$, and then

$$
\boldsymbol{B}=\lim _{n \rightarrow \infty} E\left[\frac{\partial}{\partial \boldsymbol{\lambda}} \boldsymbol{T}_{n}\right] .
$$

For our purposes we need the following consequence of Theorem 4.

TheOREM 5 (cf. [16], [17]). Let $\left(X_{1}, \ldots, X_{2 n}\right)$ be a sample with an absolutely continuous distribution function $F(x ; \boldsymbol{\lambda})$ differentiable with respect to the $m \times 1$ vector $\boldsymbol{\lambda}$. Set

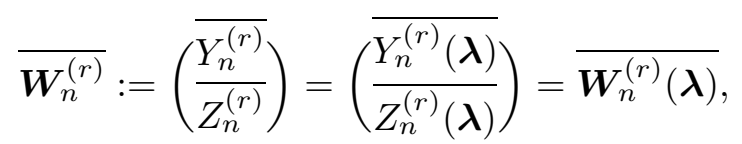

where

$$
\overline{Y_{n}^{(r)}}=\frac{1}{n} \sum_{j=1}^{2 n} F^{1+r}\left(X_{j} ; \boldsymbol{\lambda}\right), \quad \overline{Z_{n}^{(r)}}=\frac{1}{n} \sum_{j=1}^{n} F^{r}\left(X_{j}^{*} ; \boldsymbol{\lambda}\right)
$$

and

$$
X_{j}^{*}=\max \left(X_{2 j-1}, X_{2 j}\right), \quad j=1, \ldots, n .
$$

Write

$$
\widehat{\boldsymbol{W}}_{n}^{(r)}=\overline{\boldsymbol{W}_{n}^{(r)}\left(\widehat{\boldsymbol{\lambda}}_{2 n}\right)}=\left(\begin{array}{c}
\widehat{Y}_{n}^{(r)} \\
\widehat{Z}_{n}^{(r)}
\end{array}\right)
$$

where

$$
\widehat{Y}_{n}^{(r)}:=\overline{Y_{n}^{(r)}\left(\widehat{\boldsymbol{\lambda}}_{2 n}\right)}, \quad \widehat{Z}_{n}^{(r)}:=\overline{Z_{n}^{(r)}\left(\widehat{\boldsymbol{\lambda}}_{2 n}\right)} .
$$

Suppose that $F$ is such that the $M L E \widehat{\boldsymbol{\lambda}}_{2 n}$ is "regular" in the sense that

$$
\sqrt{2 n}\left(\widehat{\boldsymbol{\lambda}}_{2 n}-\boldsymbol{\lambda}\right) \stackrel{D}{\rightarrow} \mathrm{N}\left(\mathbf{0}, \mathcal{I}^{-1}\right),
$$

where $\mathcal{I}=\mathcal{I}(\boldsymbol{\lambda})$ is the expected information matrix for $\boldsymbol{\lambda}$ based on a single observation, and let $\widehat{F}\left(X_{j}\right):=F\left(X_{j} ; \widehat{\boldsymbol{\lambda}}_{2 n}\right)$. Then

$$
\sqrt{n}\left(\widehat{\boldsymbol{W}}_{n}^{(r)}-\boldsymbol{\mu}_{r}\right) \stackrel{D}{\rightarrow} \boldsymbol{W} \sim \mathrm{N}\left(\mathbf{0}, \Sigma_{r 1}\right),
$$

and

$$
\begin{aligned}
& \widehat{D}_{n r}^{(0)}=A_{r}^{(0)} \frac{1}{1-(1+r)^{2} A_{r}^{(0)} K_{r}} \cdot \frac{1}{2 n}\left(\sum_{j=1}^{2 n} \widehat{F}^{1+r}\left(X_{j}\right)-\frac{2 n}{2+r}\right)^{2} \\
& \quad \stackrel{D}{\rightarrow} \chi^{2}(1)
\end{aligned}
$$




$$
\begin{aligned}
\widehat{D}_{n r}^{(1)}= & A_{r}^{(1)} \frac{1}{n}\left(\frac{1}{r} \sum_{j=1}^{n} \widehat{F}^{r}\left(X_{j}^{*}\right)-\frac{1}{1+r} \sum_{j=1}^{2 n} \widehat{F}^{1+r}\left(X_{j}\right)\right. \\
& \left.-\frac{2 n}{r(1+r)(2+r)}\right)^{2} \stackrel{D}{\rightarrow} \chi^{2}(1)
\end{aligned}
$$

$$
\begin{aligned}
\widehat{D}_{n r}^{(2)}= & A_{r}^{(2)} \frac{2}{1-(1-r)^{2} A_{r}^{(2)} K_{r}} \cdot \frac{1}{n}\left(\sum_{j=1}^{n} \widehat{F}^{r}\left(X_{j}^{*}\right)\right. \\
& \left.-\frac{1}{2} \sum_{j=1}^{2 n} \widehat{F}^{1+r}\left(X_{j}\right)-\frac{n}{2+r}\right)^{2} \stackrel{D}{\rightarrow} \chi^{2}(1),
\end{aligned}
$$

(3.13) $\widehat{D}_{n r}^{(3)}=\widehat{D}_{n r}^{(0)}+\widehat{D}_{n r}^{(1)} \stackrel{D}{\rightarrow} \chi^{2}(2)$,

where $\Sigma_{r 1}=\Sigma_{r}-\boldsymbol{B}_{r}(2 \mathcal{I})^{-1} \boldsymbol{B}_{r}^{\prime}, \boldsymbol{\mu}_{r}$ is in (3.1), $\Sigma_{r}$ is in (3.2), $A_{r}^{(0)}, A_{r}^{(1)}$, $A_{r}^{(2)}$ are in (3.4), and

$$
\boldsymbol{B}_{r}=2\left(\begin{array}{c}
1+r \\
r
\end{array}\right) \boldsymbol{d}_{r}^{\prime}, \quad K_{r}=\boldsymbol{d}_{r}^{\prime} \mathcal{I}^{-1} \boldsymbol{d}_{r}
$$

where

$$
\boldsymbol{d}_{r}=E\left(F^{r}(X ; \boldsymbol{\lambda}) \frac{\partial F(X ; \boldsymbol{\lambda})}{\partial \boldsymbol{\lambda}}\right) \quad \text { is } m \times 1 .
$$

Proof. We apply Theorem 4 with $\boldsymbol{T}_{n}=\overline{\boldsymbol{W}_{n}^{(r)}}-\boldsymbol{\mu}_{r}$. It is known from Section 3 that

$$
\sqrt{n}\left(\overline{\boldsymbol{W}_{n}^{(r)}}-\boldsymbol{\mu}_{r}\right) \stackrel{D}{\rightarrow} \mathrm{N}\left(\mathbf{0}, \Sigma_{r}\right),
$$

and by Theorem 4 ,

$$
\sqrt{n}\left(\widehat{\boldsymbol{W}}_{n}^{(r)}-\boldsymbol{\mu}_{r}\right) \stackrel{D}{\rightarrow} \mathrm{N}\left(\mathbf{0}, \Sigma_{r 1}\right)
$$

which implies

$$
\eta_{n}=n\left(\widehat{\boldsymbol{W}}_{n}^{(r)}-\boldsymbol{\mu}_{r}\right) \Sigma_{r 1}^{-1}\left(\widehat{\boldsymbol{W}}_{n}^{(r)}-\boldsymbol{\mu}_{r}\right) \stackrel{D}{\rightarrow} \chi^{2}(2),
$$

where $\Sigma_{r 1}=\Sigma_{r}-\frac{1}{2} \boldsymbol{B}_{r} \mathcal{I}^{-1} \boldsymbol{B}_{r}$ and

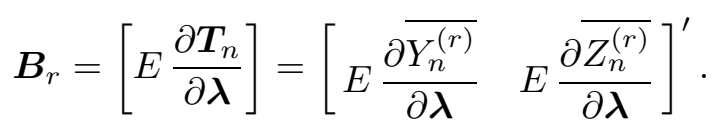

Now

and

$$
E \frac{\partial \overline{Y_{n}^{(r)}}}{\partial \lambda_{i}}=2 E \frac{\partial F^{1+r}(X ; \boldsymbol{\lambda})}{\partial \lambda_{i}}=2(1+r) E F^{r}(X ; \boldsymbol{\lambda}) \frac{\partial F(X ; \boldsymbol{\lambda})}{\partial \lambda_{i}}
$$

$$
E \frac{\partial \overline{Z_{n}^{(r)}}}{\partial \lambda_{i}}=E \frac{\partial F^{r}\left(X_{1}^{*} ; \boldsymbol{\lambda}\right)}{\partial \lambda_{i}}=r E F^{r-1}\left(X_{1}^{*} ; \boldsymbol{\lambda}\right) \frac{\partial F\left(X_{1}^{*} ; \boldsymbol{\lambda}\right)}{\partial \lambda_{i}} .
$$


But since $X_{1}^{*}$ has probability density function $2 F(x ; \boldsymbol{\lambda}) f(x ; \boldsymbol{\lambda})$, we have

$$
E \frac{\partial \overline{Z_{n}^{(r)}}}{\partial \lambda_{i}}=2 r \int F^{r}(x ; \boldsymbol{\lambda}) \frac{\partial F(x ; \boldsymbol{\lambda})}{\partial \lambda_{i}} f(x ; \boldsymbol{\lambda}) d x=\frac{2 r}{1+r} E \frac{\partial \overline{Y_{n}^{(r)}}}{\partial \lambda_{i}} .
$$

It follows that $\boldsymbol{B}_{r}^{\prime}=\boldsymbol{d}_{r} \boldsymbol{b}_{r}^{\prime}$ where

$$
\boldsymbol{d}_{r}=E\left(F^{r}(X ; \boldsymbol{\lambda}) \frac{\partial F(X ; \boldsymbol{\lambda})}{\partial \boldsymbol{\lambda}}\right), \quad \boldsymbol{b}_{r}^{\prime}=2[1+r \quad r]
$$

and

(3.16) $\quad \Sigma_{r 1}=\Sigma_{r}-\frac{1}{2} \boldsymbol{b}_{r} \boldsymbol{d}_{r}^{\prime} \mathcal{I}^{-1} \boldsymbol{d}_{r} \boldsymbol{b}_{r}^{\prime}=\Sigma_{r}-2 K_{r}\left[\begin{array}{cc}(1+r)^{2} & r(1+r) \\ r(1+r) & r^{2}\end{array}\right]$.

Now write

$$
\boldsymbol{A}=\left[\begin{array}{cc}
1 & 0 \\
-\frac{r}{1+r} & 1
\end{array}\right]
$$

and consider $\boldsymbol{U}_{n}=\boldsymbol{A}\left(\overline{\boldsymbol{W}_{n}^{(r)}}-\boldsymbol{\mu}_{r}\right)$. Then

$$
\boldsymbol{U}_{n}=\left[\begin{array}{c}
\overline{Y_{n}^{(r)}} \\
\overline{Z_{n}^{(r)}}-\frac{r}{1+r} \overline{Y_{n}^{(r)}}
\end{array}\right]-2\left[\begin{array}{c}
\frac{1}{2+r} \\
\frac{1}{(1+r)(2+r)}
\end{array}\right],
$$

and from (3.14) and (3.15),

$$
\eta_{n}=n \boldsymbol{U}_{n}^{\prime}\left(\boldsymbol{A} \Sigma_{r 1} \boldsymbol{A}^{\prime}\right)^{-1} \boldsymbol{U}_{n} .
$$

Now from (3.16),

$$
\boldsymbol{A} \Sigma_{r 1} \boldsymbol{A}^{\prime}=\boldsymbol{A} \Sigma_{r} \boldsymbol{A}^{\prime}-2 K_{r}\left(\boldsymbol{A} \boldsymbol{b}_{r}\right)\left(\boldsymbol{A} \boldsymbol{b}_{r}\right)^{\prime} .
$$

But

$$
\boldsymbol{A b}_{r}=2(1+r)\left[\begin{array}{ll}
1 & 0
\end{array}\right]^{\prime},
$$

and one finds that $\boldsymbol{A} \Sigma_{r 1} \boldsymbol{A}^{\prime}$ simplifies to

$$
\left[\begin{array}{cc}
2\left(1-(2+r)^{2}(3+2 r)\right) K_{r} / A_{r}^{(0)} & 0 \\
0 & r^{2} / A_{r}^{(1)}
\end{array}\right] .
$$

It then follows that

$$
\eta_{n}=\widehat{D}_{n r}^{(0)}+\widehat{D}_{n r}^{(1)}=\widehat{D}_{n r}^{(3)}
$$

which proves (3.13).

Finally, (3.11) and (3.12) follow from the fact that since

$$
\sqrt{n} \boldsymbol{U}_{n} \stackrel{D}{\rightarrow} N\left(\mathbf{0}, A \Sigma_{r 1} A^{\prime}\right)
$$

we have

$$
\sqrt{n}\left[\overline{Y_{n}^{(r)}}-\frac{2}{2+r}\right] \stackrel{D}{\rightarrow} \mathrm{N}\left(0,2\left(1-(2+r)^{2}(3+2 r)\right) K_{r} / A_{r}^{(0)}\right)
$$


and

$$
\sqrt{n}\left[\overline{Z_{n}^{(r)}}-\frac{r}{r+1} \overline{Y_{n}^{(r)}}-\frac{2}{(1+r)(2+r)}\right] \stackrel{D}{\rightarrow} \mathrm{N}\left(0, r^{2} / A_{r}^{(1)}\right) .
$$

To establish $\left(3.12^{\prime}\right)$, consider

$$
S_{n r}=\widehat{Z}_{n}^{(r)}-\frac{1}{2} \widehat{Y}_{n}^{(r)}=\boldsymbol{\alpha}^{\prime} \widehat{\boldsymbol{W}}_{n}^{(r)}
$$

where $\boldsymbol{\alpha}^{\prime}=\left[\begin{array}{ll}-\frac{1}{2} & 1\end{array}\right]$. Then

$$
\sqrt{n}\left[S_{n r}-\frac{1}{2+r}\right]=\sqrt{n} \boldsymbol{\alpha}^{\prime}\left[\widehat{\boldsymbol{W}}_{n}^{(r)}-\mu_{r}\right] \stackrel{D}{\rightarrow} \mathrm{N}\left(0, \sigma_{n r}^{2}\right)
$$

where

$$
\sigma_{n r}^{2}=\boldsymbol{\alpha}^{\prime} \Sigma_{r 1} \boldsymbol{\alpha}=\boldsymbol{\alpha}^{\prime} \Sigma_{r} \boldsymbol{\alpha}-\frac{1}{2} K_{r}\left[\boldsymbol{\alpha}^{\prime} \boldsymbol{b}_{r}\right]^{2}=\frac{1}{A_{r}^{(2)}}-\frac{1}{2} K_{r}(1-r)^{2} .
$$

Thus

$$
n\left(S_{n r}-\frac{1}{2+r}\right)^{2} / \sigma_{n r}^{2} \stackrel{D}{\rightarrow} \chi^{2}(1),
$$

which proves $\left(3.12^{\prime}\right)$.

Note. Since $\boldsymbol{d}_{r}$ and $\mathcal{I}$ may depend on $\boldsymbol{\lambda}, K_{r}$ may also depend on $\boldsymbol{\lambda}$. In this case $\widehat{D}_{n r}^{(0)}, \widehat{D}_{n r}^{(2)}$ and $\widehat{D}_{n r}^{(3)}$ also depend on $\boldsymbol{\lambda}$, and cannot be used as test-statistics. But if $K_{r}=K_{r}(\boldsymbol{\lambda})$ is replaced by $\widehat{K}_{r}=K_{r}\left(\widehat{\boldsymbol{\lambda}}_{2 n}\right)$ the resulting statistic satisfies $\widehat{D}_{n r}^{(0)}\left(\widehat{\boldsymbol{\lambda}}_{2 n}\right) \stackrel{D}{\rightarrow} \chi^{2}(1)$, and similarly for $\widehat{D}_{n r}^{(2)}$ and $\widehat{D}_{n r}^{(3)}$. This follows because

$$
\widehat{D}_{n r}^{(0)}\left(\widehat{\boldsymbol{\lambda}}_{2 n}\right)=\widehat{D}_{n r}^{(0)}(\boldsymbol{\lambda}) \frac{1-(2+r)^{2}(3+2 r) K_{r}(\boldsymbol{\lambda})}{1-(2+r)^{2}(3+2 r) K_{r}\left(\widehat{\boldsymbol{\lambda}}_{2 n}\right)}
$$

and

$$
\frac{1-(2+r)^{2}(3+2 r) K_{r}(\boldsymbol{\lambda})}{1-(2+r)^{2}(3+2 r) K_{r}\left(\widehat{\boldsymbol{\lambda}}_{2 n}\right)} \stackrel{P}{\rightarrow} 1
$$

since $\widehat{\boldsymbol{\lambda}}_{2 n} \stackrel{P}{\rightarrow} \boldsymbol{\lambda}$.

Corollary 5 (cf. [17]). If $r=1$ then

$$
\begin{aligned}
\text { (3.17) } \widehat{D}_{n 1}^{(0)} & =\frac{45}{4} \cdot \frac{1}{\left(1-45 K_{1}\right)} \cdot \frac{1}{2 n}\left(\sum_{j=1}^{2 n} \widehat{F}^{2}\left(X_{j}\right)-\frac{2}{3} n\right)^{2} \stackrel{D}{\rightarrow} \chi^{2}(1), \\
\text { (3.18) } \widehat{D}_{n 1}^{(1)} & =\widehat{D}_{n 1}^{(2)}=90 \frac{1}{n}\left(\sum_{j=1}^{n} \widehat{F}\left(X_{j}^{*}\right)-\frac{1}{2} \sum_{j=1}^{2 n} \widehat{F}^{2}\left(X_{j}\right)-\frac{1}{3} n\right)^{2} \\
& \stackrel{D}{\rightarrow} \chi^{2}(1), \\
\text { (3.19) } \widehat{D}_{n 1}^{(3)} & =\widehat{D}_{n 1}^{(0)}+\widehat{D}_{n 1}^{(1)} \stackrel{D}{\rightarrow} \chi^{2}(2) .
\end{aligned}
$$


Now we present tests based on Theorem 5 and Corollary 5 for special distributions.

$1^{\circ}$. Let $F \in \operatorname{Exp}(\alpha)$, i.e. $F(x)=1-e^{-\alpha x}, f(x)=\alpha e^{-\alpha x}$. We see that

$$
\begin{aligned}
d_{r}(\alpha) & =E F^{r}(X) \frac{\partial F}{\partial \alpha}=E\left(1-e^{-\alpha X}\right)^{r} X e^{-\alpha X} \\
& =\alpha \int_{0}^{\infty}\left(1-e^{-\alpha x}\right)^{r} x e^{-2 \alpha x} d x=\alpha \int_{0}^{\infty}\left(\sum_{j=0}^{\infty}(-1)^{j}\left(\begin{array}{l}
r \\
j
\end{array}\right) e^{-j \alpha x}\right) x e^{-2 \alpha x} d x \\
& =\frac{1}{\alpha} \sum_{j=0}^{\infty}(-1)^{j}\left(\begin{array}{l}
r \\
j
\end{array}\right) \frac{1}{(j+2)^{2}},
\end{aligned}
$$

and

$$
\mathcal{I}^{-1}=\alpha^{2}
$$

Then letting

$$
S_{r}=\sum_{j=0}^{\infty}(-1)^{j}\left(\begin{array}{l}
r \\
j
\end{array}\right) \frac{1}{(j+2)^{2}}
$$

we obtain $K_{r}=S_{r}^{2}$. Therefore we have

Proposition 4. Goodness-of-fit tests for $F \in \operatorname{Exp}(\alpha)$ are given by

$$
\begin{aligned}
\widehat{D}_{n r}^{(0)}= & \frac{(2+r)^{2}(3+2 r)}{(1+r)^{2}\left(1-(2+r)^{2}(3+2 r) S_{r}^{2}\right)} \\
& \times \frac{1}{2 n}\left(\sum_{j=1}^{2 n}\left(1-e^{-\widehat{\alpha}_{2 n} X_{j}}\right)^{1+r}-\frac{2 n}{2+r}\right)^{2} \stackrel{D}{\rightarrow} \chi^{2}(1), \\
\widehat{D}_{n r}^{(1)}= & (1+r)(2+r)^{2}(3+2 r) \frac{1}{n}\left(\frac{1}{r} \sum_{j=1}^{n}\left(1-e^{-\widehat{\alpha}_{2 n} X_{j}^{*}}\right)^{r}\right. \\
& \left.-\frac{1}{1+r} \sum_{j=1}^{2 n}\left(1-e^{-\widehat{\alpha}_{2 n} X_{j}}\right)^{1+r}-\frac{2 n}{r(1+r)(2+r)}\right)^{2} \stackrel{D}{\rightarrow} \chi^{2}(1), \\
\widehat{D}_{n r}^{(2)}= & \frac{2(1+r)(2+r)^{2}(3+2 r)}{r^{3}+r^{2}-r+1-(1-r)^{2}(1+r)(2+r)^{2}(3+2 r) S_{r}^{2}} \\
& \times \frac{1}{n}\left(\sum_{j=1}^{n}\left(1-e^{-\widehat{\alpha}_{2 n} X_{j}^{*}}\right)^{r}-\frac{1}{2} \sum_{j=1}^{2 n}\left(1-e^{-\widehat{\alpha}_{2 n} X_{j}}\right)^{1+r}-\frac{n}{2+r}\right)^{2} \\
\stackrel{D}{\rightarrow} & \chi^{2}(1), \\
\widehat{D}_{n r}^{(3)}= & \widehat{D}_{n r}^{(0)}+\widehat{D}_{n r}^{(1)} \stackrel{D}{\rightarrow} \chi^{2}(2),
\end{aligned}
$$

where $A_{r}^{(0)}, A_{r}^{(1)}, A_{r}^{(2)}$ are in (3.4), and $\widehat{\alpha}_{2 n}=1 / \bar{X}_{2 n}$. 
REMARK. If $r$ is a positive integer then

$$
S_{r}=\sum_{j=0}^{r}(-1)^{j}\left(\begin{array}{l}
r \\
j
\end{array}\right) \frac{1}{(j+2)^{2}} .
$$

Numerical evaluation of $K_{r}$

\begin{tabular}{llllll}
\hline$r$ & 0.001 & 0.010 & 0.050 & 0.10 & 0.20 \\
$K_{r}$ & 0.06241 & 0.06162 & 0.05826 & 0.05439 & 0.04761 \\
\hline$r$ & 0.25 & 0.30 & 0.40 & 0.50 & 0.60 \\
$K_{r}$ & 0.04464 & 0.04190 & 0.03705 & 0.03292 & 0.02937 \\
\hline$r$ & 0.70 & 0.75 & 0.80 & 0.90 & 1.00 \\
$K_{r}$ & 0.02630 & 0.02492 & 0.02364 & 0.02132 & 0.01929 \\
\hline$r$ & 1.25 & 1.50 & 1.75 & 2.00 & 2.50 \\
$K_{r}$ & 0.015212 & 0.012190 & 0.009907 & 0.008150 & 0.005692 \\
\hline$r$ & 3.00 & 3.50 & 4.00 & 4.50 & 5.00 \\
$K_{r}$ & 0.004117 & 0.0030650 & 0.0023361 & 0.0018172 & 0.0014383 \\
\hline
\end{tabular}

$2^{\text {o. Let }} F \in \mathrm{W}(\alpha, \beta)$, i.e. $F(x)=1-\exp \left(-\alpha x^{\beta}\right), f(x)=\alpha \beta x^{\beta-1} e^{-\alpha x^{\beta}}$. We see that

$$
\frac{\partial F}{\partial \alpha}=\frac{x}{\alpha \beta} f(x), \quad \frac{\partial F}{\partial \beta}=\frac{x \ln x}{\beta} f(x) .
$$

Hence we have

$$
\begin{aligned}
d_{r}(\alpha) & =E F^{r}(X) \frac{\partial F}{\partial \alpha}=\int_{0}^{\infty}\left(1-\exp \left(-\alpha x^{\beta}\right)\right)^{r} \frac{x}{\alpha \beta} f^{2}(x) d x \\
& =\sum_{j=0}^{\infty}(-1)^{j}\left(\begin{array}{l}
r \\
j
\end{array}\right) \frac{1}{j+2} \int_{0}^{\infty} x^{\beta} \alpha(j+2) \beta x^{\beta-1} e^{-(j+2) \alpha x^{\beta}} d x \\
& =\sum_{j=0}^{\infty}(-1)^{j}\left(\begin{array}{l}
r \\
j
\end{array}\right) \frac{1}{j+2} E X_{j}^{\beta},
\end{aligned}
$$

where $X_{j} \sim \mathrm{W}((j+2) \alpha, \beta)$, and

$$
\begin{aligned}
d_{r}(\beta) & =E F^{r}(X) \frac{\partial F}{\partial \beta}=\int_{0}^{\infty}\left(1-\exp \left(-\alpha x^{\beta}\right)\right)^{r} \frac{x \ln x}{\beta} f^{2}(x) d x \\
& =\alpha \sum_{j=0}^{\infty}(-1)^{j}\left(\begin{array}{l}
r \\
j
\end{array}\right) \frac{1}{j+2} E X_{j}^{\beta} \ln X_{j} .
\end{aligned}
$$

But for $X \sim \mathrm{W}(\alpha, \beta)$ we have

$$
\begin{aligned}
E X^{\beta} & =\alpha \beta \int_{0}^{\infty} x^{2 \beta-1} e^{-\alpha x^{\beta}} d x=1 / \alpha \\
E X^{\beta} \ln X & =\alpha \beta \int_{0}^{\infty} x^{2 \beta-1} \ln x e^{-\alpha x^{\beta}} d x=\frac{1}{\alpha \beta} \int_{0}^{\infty}(\ln y-\ln \alpha) y e^{-y} d y
\end{aligned}
$$




$$
\begin{aligned}
& =\frac{1}{\alpha \beta} \int_{0}^{\infty} y \ln y e^{-y} d y-\frac{\ln \alpha}{\alpha \beta} \\
& =\frac{1}{\alpha \beta}\left(\int_{0}^{\infty} e^{-y} \ln y d y+\int_{0}^{\infty} e^{-y} d y\right)-\frac{\ln \alpha}{\alpha \beta} \\
& =\frac{1}{\alpha \beta}(1-\gamma-\ln \alpha),
\end{aligned}
$$

where $\gamma=-\int_{0}^{\infty} e^{-y} \ln y d y$ (cf. [22, 3.711.2]) is the Euler constant. Moreover,

$$
\begin{aligned}
E X^{\beta} \ln ^{2} X & =\alpha \beta \int_{0}^{\infty} x^{2 \beta-1} \ln ^{2} x e^{-\alpha x^{\beta}} d x \\
& =\frac{1}{\alpha \beta^{2}} \int_{0}^{\infty}(\ln y-\ln \alpha)^{2} y e^{-y} d y \\
& =\frac{1}{\alpha \beta^{2}} \int_{0}^{\infty} e^{-y} y \ln ^{2} y d y-\frac{2 \ln \alpha}{\alpha \beta^{2}} \int_{0}^{\infty} e^{-y} y \ln y d y+\frac{\ln ^{2} \alpha}{\alpha \beta^{2}} \\
& =\frac{1}{\alpha \beta^{2}} \int_{0}^{\infty} e^{-y} y \ln ^{2} y d y-\frac{2 \ln \alpha}{\alpha \beta^{2}}(1-\gamma)+\frac{\ln ^{2} \alpha}{\alpha \beta^{2}} \\
& =\frac{1}{\alpha \beta^{2}} \int_{0}^{\infty} e^{-y} \ln ^{2} y d y+\frac{2}{\alpha \beta^{2}} \int_{0}^{\infty} e^{-y} \ln y d y \\
& -\frac{2 \ln \alpha}{\alpha \beta^{2}}(1-\gamma)+\frac{\ln ^{2} \alpha}{\alpha \beta^{2}} \\
& =\frac{1}{\alpha \beta^{2}} \int_{0}^{\infty} e^{-y} \ln ^{2} y d y-\frac{2}{\alpha \beta^{2}} \gamma-\frac{2 \ln \alpha}{\alpha \beta^{2}}(1-\gamma)+\frac{\ln ^{2} \alpha}{\alpha \beta^{2}} \\
& =\frac{1}{\alpha \beta^{2}}\left[(1-\gamma-\ln \alpha)^{2}+\frac{\pi^{2}}{6}-1\right],
\end{aligned}
$$

where the formula

$$
\int_{0}^{\infty} e^{-x} \ln ^{2} x d x=\Gamma^{\prime \prime}(1)=\gamma^{2}+\frac{1}{6} \pi^{2} \quad(\text { cf. }[22,3.714 .1])
$$

has been used. Thus

$$
\begin{gathered}
E X_{j}^{\beta}=\frac{1}{\alpha(j+2)}, \quad E X_{j}^{\beta} \ln X_{j}=\frac{1}{\alpha(j+2) \beta}[1-\gamma-\ln \alpha(j+2)], \\
E X_{j}^{\beta} \ln ^{2} X_{j}=\frac{1}{\alpha(j+2) \beta^{2}}\left[(1-\gamma-\ln \alpha(j+2))^{2}+\frac{\pi^{2}}{6}-1\right] .
\end{gathered}
$$


Hence

$$
\begin{aligned}
d_{r}(\alpha) & =\sum_{j=0}^{\infty}(-1)^{j}\left(\begin{array}{l}
r \\
j
\end{array}\right) \frac{1}{j+2} E X_{j}^{\beta}=\frac{1}{\alpha} S_{r}, \\
d_{r}(\beta) & =\alpha \sum_{j=0}^{\infty}(-1)^{j}\left(\begin{array}{l}
r \\
j
\end{array}\right) \frac{1}{j+2} E X_{j}^{\beta} \ln X_{j} \\
& =\frac{1}{\beta} \sum_{j=0}^{\infty}(-1)^{j}\left(\begin{array}{l}
r \\
j
\end{array}\right) \frac{1}{(j+2)^{2}}[1-\gamma-\ln \alpha(j+2)] \\
& =\frac{1-\gamma-\ln \alpha}{\beta} \sum_{j=0}^{\infty}(-1)^{j}\left(\begin{array}{l}
r \\
j
\end{array}\right) \frac{1}{(j+2)^{2}}-\frac{1}{\beta} \sum_{j=0}^{\infty}(-1)^{j}\left(\begin{array}{l}
r \\
j
\end{array}\right) \frac{\ln (j+2)}{(j+2)^{2}} \\
& =\frac{1}{\beta}\left[(1-\gamma-\ln \alpha) S_{r}-T_{r}\right],
\end{aligned}
$$

where

$$
T_{r}=\sum_{j=0}^{\infty}(-1)^{j}\left(\begin{array}{l}
r \\
j
\end{array}\right) \frac{\ln (j+2)}{(j+2)^{2}} .
$$

Moreover, we have

$$
\begin{aligned}
\mathcal{I} & =\left[\begin{array}{cc}
1 / \alpha^{2} & E X^{\beta} \ln X \\
E X^{\beta} \ln X & 1 / \beta^{2}+\alpha E X^{\beta} \ln ^{2} X
\end{array}\right] \\
& =\left[\begin{array}{cc}
1 / \alpha^{2} & \frac{1}{\alpha \beta}[1-\gamma-\ln \alpha] \\
\frac{1}{\alpha \beta}[1-\gamma-\ln \alpha] & \frac{1}{\beta^{2}}\left[(1-\gamma-\ln \alpha)^{2}+\pi^{2} / 6\right]
\end{array}\right], \\
\mathcal{I}^{-1} & =\frac{6}{\pi^{2}}\left[\begin{array}{cc}
\alpha^{2}\left(\pi^{2} / 6+(1-\gamma-\ln \alpha)^{2}\right) & -\alpha \beta(1-\gamma-\ln \alpha) \\
-\alpha \beta(1-\gamma-\ln \alpha) & \beta^{2}
\end{array}\right]
\end{aligned}
$$

(cf. [10, p. 256]). Therefore

$$
\boldsymbol{d}_{r}=\left(\begin{array}{c}
\frac{1}{\alpha} S_{r} \\
\frac{1}{\beta}\left[(1-\gamma-\ln \alpha) S_{r}-T_{r}\right]
\end{array}\right)
$$

and

$$
K_{r}=\boldsymbol{d}_{r}^{\prime} \mathcal{I}^{-1} \boldsymbol{d}_{r}=S_{r}^{2}+\frac{6}{\pi^{2}} T_{r}^{2}
$$

Thus we have

Proposition 5. Goodness-of-fit tests for $F \in \mathrm{W}(\alpha, \beta)$ are given by

$$
\begin{aligned}
\widehat{D}_{n r}^{(0)}= & \frac{(2+r)^{2}(3+2 r)}{(1+r)^{2}\left\{1-(2+r)^{2}(3+2 r)\left[S_{r}^{2}+\frac{6}{\pi^{2}} T_{r}^{2}\right]\right\}} \\
& \times \frac{1}{2 n}\left\{\sum_{j=1}^{2 n}\left(1-\exp \left(-\widehat{\alpha}_{2 n} X_{j}^{\widehat{\beta}_{2 n}}\right)\right)^{1+r}-\frac{2 n}{2+r}\right\}^{2} \stackrel{D}{\rightarrow} \chi^{2}(1),
\end{aligned}
$$




$$
\begin{aligned}
\widehat{D}_{n r}^{(1)}= & (1+r)(2+r)^{2}(3+2 r) \frac{1}{n}\left(\frac { 1 } { r } \sum _ { j = 1 } ^ { n } \left(1-\exp \left(-\widehat{\alpha}_{2 n}\left(X_{j}^{*} \widehat{\beta}_{2 n}\right)\right)^{r}\right.\right. \\
& \left.-\frac{1}{1+r} \sum_{j=1}^{2 n}\left(1-\exp \left(-\widehat{\alpha}_{2 n} X_{j}^{\widehat{\beta}_{2 n}}\right)\right)^{1+r}-\frac{2 n}{r(1+r)(2+r)}\right)^{2} \stackrel{D}{\rightarrow} \chi^{2}(1), \\
\widehat{D}_{n r}^{(2)}= & \frac{2(1+r)(2+r)^{2}(3+2 r)}{r^{3}+r^{2}-r+1-(1-r)^{2}(1+r)(2+r)^{2}(3+2 r)\left[S_{r}^{2}+\frac{6}{\pi^{2}} T_{r}^{2}\right]} \\
& \times \frac{1}{n}\left(\sum_{j=1}^{n}\left(1-\exp \left(-\widehat{\alpha}_{2 n}\left(X_{j}^{*}\right)^{\widehat{\beta}_{2 n}}\right)\right)^{r}\right. \\
& \left.-\frac{1}{2} \sum_{j=1}^{2 n}\left(1-\exp \left(-\widehat{\alpha}_{2 n} X_{j}^{\widehat{\beta}_{2 n}}\right)\right)^{1+r}-\frac{n}{2+r}\right)^{2} \stackrel{D}{\rightarrow} \chi^{2}(1) \\
\widehat{D}_{n r}^{(3)}= & \widehat{D}_{n r}^{(0)}+\widehat{D}_{n r}^{(1)} \stackrel{D}{\rightarrow} \chi^{2}(2),
\end{aligned}
$$

where

$$
\widehat{\alpha}_{2 n}=2 n / \sum_{i=1}^{2 n} X_{i}^{\widehat{\beta}_{2 n}}
$$

and $\widehat{\beta}_{2 n}$ is obtained by numerical solution of the equation

$$
\frac{d}{d \beta} L_{2 n}\left(2 n / \sum_{i=1}^{2 n} x_{i}^{\beta}, \beta\right)=0
$$

for

$$
L_{2 n}(\alpha, \beta)=2 n \ln \alpha+2 n \ln \beta+(\beta-1) \sum_{i=1}^{2 n} \ln x_{i}-\alpha \sum_{i=1}^{2 n} x_{i}^{\beta}
$$

Numerical evaluation of $K_{r}$

\begin{tabular}{llllll}
\hline$r$ & 0.001 & 0.010 & 0.050 & 0.10 & 0.20 \\
$K_{r}$ & 0.08062 & 0.07939 & 0.07423 & 0.06838 & 0.05840 \\
\hline$r$ & 0.25 & 0.30 & 0.40 & 0.50 & 0.60 \\
$K_{r}$ & 0.05414 & 0.05028 & 0.04360 & 0.03805 & 0.03340 \\
\hline$r$ & 0.70 & 0.75 & 0.80 & 0.90 & 1.00 \\
$K_{r}$ & 0.02949 & 0.02776 & 0.02617 & 0.02333 & 0.02088 \\
\hline$r$ & 1.25 & 1.50 & 1.75 & 2.00 & 2.50 \\
$K_{r}$ & 0.016113 & 0.012698 & 0.010188 & 0.008302 & 0.005729 \\
\hline$r$ & 3.00 & 3.50 & 4.00 & 4.50 & 5.00 \\
$K_{r}$ & 0.004122 & 0.003065 & 0.0023411 & 0.0018285 & 0.0014551 \\
\hline
\end{tabular}

$3^{o}$. Let $F \in \operatorname{Par}_{\mathrm{S}}(\alpha, \sigma)$, i.e. $F(x)=1-(\sigma / x)^{\alpha}, f(x)=\alpha \sigma^{\alpha} / x^{\alpha+1}$.

We consider first the case when $\sigma$ is known, which frequently occurs in practice. Since $Y=\ln (X / \sigma) \sim \operatorname{Exp}(\alpha)$, Proposition 4 yields 
Proposition 6. Goodness-of-fit tests for $F \in \operatorname{Par}_{\mathrm{S}}(\alpha, \sigma)$ when $\sigma$ is known are given by

$$
\begin{aligned}
\widehat{D}_{n r}^{(0)}= & \frac{(2+r)^{2}(3+2 r)}{(1+r)^{2}\left(1-(2+r)^{2}(3+2 r) S_{r}^{2}\right)} \\
& \times \frac{1}{2 n}\left(\sum_{j=1}^{2 n}\left(1-e^{-\widehat{\alpha}_{2 n} Y_{j}}\right)^{1+r}-\frac{2 n}{2+r}\right)^{2} \stackrel{D}{\rightarrow} \chi^{2}(1), \\
\widehat{D}_{n r}^{(1)}= & (1+r)(2+r)^{2}(3+2 r) \frac{1}{n}\left(\frac{1}{r} \sum_{j=1}^{n}\left(1-e^{-\widehat{\alpha}_{2 n} Y_{j}^{*}}\right)^{r}\right. \\
& \left.-\frac{1}{1+r} \sum_{j=1}^{2 n}\left(1-e^{-\widehat{\alpha}_{2 n} Y_{j}}\right)^{1+r}-\frac{2 n}{r(1+r)(2+r)}\right)^{2} \stackrel{D}{\rightarrow} \chi^{2}(1), \\
\widehat{D}_{n r}^{(2)}= & \frac{2(1+r)(2+r)^{2}(3+2 r)}{r^{3}+r^{2}-r+1-(1-r)^{2}(1+r)(2+r)^{2}(3+2 r) S_{r}^{2}} \\
& \times \frac{1}{n}\left(\sum_{j=1}^{n}\left(1-e^{-\widehat{\alpha}_{2 n} Y_{j}^{*}}\right)^{r}-\frac{1}{2} \sum_{j=1}^{2 n}\left(1-e^{-\widehat{\alpha}_{2 n} Y_{j}}\right)^{1+r}-\frac{n}{2+r}\right)^{2} \\
\stackrel{D}{\rightarrow} & \chi^{2}(1), \\
\widehat{D}_{n r}^{(3)}= & \widehat{D}_{n r}^{(0)}+\widehat{D}_{n r}^{(1)} \stackrel{D}{\rightarrow} \chi^{2}(2),
\end{aligned}
$$

where

$$
Y_{j}^{*}=\max \left(Y_{2 j-1}, Y_{2 j}\right), \quad j=1, \ldots, n, \quad \frac{1}{\widehat{\alpha}_{2 n}}=\frac{1}{2 n} \sum_{j=1}^{2 n} \ln \left(\frac{X_{j}}{\sigma}\right) .
$$

When both $\sigma$ and $\alpha$ are unknown we cannot apply Theorem 5 since this is a situation where the MLE are not regular. But then we can use

Proposition 6a. Goodness-of-fit tests for $F \in \operatorname{Par}_{S}(\alpha, \sigma)$ when $\alpha$ and $\sigma$ are unknown are given by

$$
\begin{aligned}
\widehat{\widehat{D}}_{n r}^{(0)}= & \frac{(2+r)^{2}(3+2 r)}{(1+r)^{2}\left(1-(2+r)^{2}(3+2 r) S_{r}^{2}\right)} \\
& \times \frac{1}{2 n}\left(\sum_{j=1}^{2 n}\left(1-e^{-\widehat{\hat{\alpha}}_{2 n} \widehat{Y}_{j}}\right)^{1+r}-\frac{2 n}{2+r}\right)^{2} \stackrel{D}{\rightarrow} \chi^{2}(1), \\
\widehat{\widehat{D}}_{n r}^{(1)}= & (1+r)(2+r)^{2}(3+2 r) \frac{1}{n}\left(\frac{1}{r} \sum_{j=1}^{n}\left(1-e^{-\widehat{\hat{\alpha}}_{2 n} \widehat{Y}_{j}^{*}}\right)^{r}\right. \\
& \left.-\frac{1}{1+r} \sum_{j=1}^{2 n}\left(1-e^{-\widehat{\hat{\alpha}}_{2 n} \widehat{Y}_{j}}\right)^{1+r}-\frac{2 n}{r(1+r)(2+r)}\right)^{2} \stackrel{D}{\rightarrow} \chi^{2}(1),
\end{aligned}
$$




$$
\begin{aligned}
\widehat{\widehat{D}}_{n r}^{(2)}= & \frac{2(1+r)(2+r)^{2}(3+2 r)}{r^{3}+r^{2}-r+1-(1-r)^{2}(1+r)(2+r)^{2}(3+2 r) S_{r}^{2}} \\
& \times \frac{1}{n}\left(\sum_{j=1}^{n}\left(1-e^{-\widehat{\hat{\alpha}}_{2 n} \widehat{Y}_{j}^{*}}\right)^{r}-\frac{1}{2} \sum_{j=1}^{2 n}\left(1-e^{-\widehat{\hat{\alpha}}_{2 n} \widehat{Y}_{j}}\right)^{1+r}-\frac{n}{2+r}\right)^{2} \\
& \stackrel{D}{\rightarrow} \chi^{2}(1), \\
\widehat{\widehat{D}}_{n r}^{(3)}= & \widehat{\widehat{D}}_{n r}^{(0)}+\widehat{\widehat{D}}_{n r}^{(1)} \stackrel{D}{\rightarrow} \chi^{2}(2),
\end{aligned}
$$

where

$$
\begin{gathered}
\widehat{Y}_{j}=\ln \left(X_{j} / \widehat{\sigma}_{2 n}\right), \quad \widehat{Y}_{j}^{*}=\max \left(\widehat{Y}_{2 j-1}, \widehat{Y}_{2 j}\right), \quad j=1, \ldots, n, \\
\widehat{\sigma}_{2 n}=\min \left(X_{1}, \ldots, X_{2 n}\right), \quad 1 / \widehat{\widehat{\alpha}}_{2 n}=\frac{1}{2 n} \sum_{j=1}^{2 n} \ln \left(X_{j} / \widehat{\sigma}_{2 n}\right) .
\end{gathered}
$$

Proof. This follows from the fact that $\sqrt{2 n}\left(\widehat{\sigma}_{2 n}-\sigma\right) \stackrel{P}{\rightarrow} 0$ because then $\widehat{D}_{n r}^{(i)}-\widehat{\widehat{D}}_{n r}^{(i)} \stackrel{P}{\rightarrow} 0, i=0,1,2$.

$4^{\mathrm{o}}$. Let $F \in \operatorname{Par}_{\mathrm{T}}(\alpha, \theta)$, i.e.

$$
F(x)=1-\left(\frac{\theta}{x+\theta}\right)^{\alpha}, \quad f(x)=\frac{\alpha \theta^{\alpha}}{(x+\theta)^{\alpha+1}} .
$$

Then

$$
\frac{\partial F}{\partial \alpha}=-\left(\frac{\theta}{x+\theta}\right)^{\alpha} \ln \left(\frac{\theta}{x+\theta}\right), \quad \frac{\partial F}{\partial \theta}=-\frac{x}{\theta} f(x)
$$

Hence

$$
\begin{aligned}
d_{r}(\alpha) & =E F^{r}(X) \frac{\partial F}{\partial \alpha} \\
& =-\int_{0}^{\infty}\left(1-\left(\frac{\theta}{x+\theta}\right)^{\alpha}\right)^{r}\left(\frac{\theta}{x+\theta}\right)^{\alpha} \frac{\alpha \theta^{\alpha}}{(x+\theta)^{\alpha+1}} \ln \left(\frac{\theta}{x+\theta}\right) d x \\
& =-\frac{\alpha}{\theta} \int_{0}^{\infty}\left(1-\left(\frac{\theta}{x+\theta}\right)^{\alpha}\right)^{r}\left(\frac{\theta}{x+\theta}\right)^{2 \alpha+1} \ln \left(\frac{\theta}{x+\theta}\right) d x \\
& =-\frac{\alpha}{\theta} \sum_{j=0}^{\infty}(-1)^{j}\left(\begin{array}{l}
r \\
j
\end{array}\right) \int_{0}^{\infty}\left(\frac{\theta}{x+\theta}\right)^{j \alpha+2 \alpha+1} \ln \left(\frac{\theta}{x+\theta}\right) d x \\
& =-\alpha \sum_{j=0}^{\infty}(-1)^{j}\left(\begin{array}{l}
r \\
j
\end{array}\right) \int_{0}^{1} y^{(j+2) \alpha-1} \ln y d y \\
& =\frac{1}{\alpha} \sum_{j=0}^{\infty}(-1)^{j}\left(\begin{array}{l}
r \\
j
\end{array}\right) \frac{1}{(j+2)^{2}}=\frac{1}{\alpha} S_{r},
\end{aligned}
$$


and

$$
\begin{aligned}
d_{r}(\theta) & =E F^{r}(X) \frac{\partial F}{\partial \theta}=-\int_{0}^{\infty}\left(1-\left(\frac{\theta}{x+\theta}\right)^{\alpha}\right)^{r} \frac{x}{\theta} f^{2}(x) d x \\
& =-\frac{1}{\theta} \int_{0}^{\infty} x\left(1-\left(\frac{\theta}{x+\theta}\right)^{\alpha}\right)^{r}\left(\frac{\alpha \theta^{\alpha}}{(x+\theta)^{\alpha+1}}\right)^{2} d x \\
& =-\frac{\alpha}{\theta} \sum_{j=0}^{\infty}(-1)^{j}\left(\begin{array}{l}
r \\
j
\end{array}\right) \frac{1}{(j+2)((j+2) \alpha+1)}=-\frac{\alpha}{\theta} S_{r \alpha}
\end{aligned}
$$

where

$$
S_{r \alpha}=\sum_{j=0}^{\infty}(-1)^{j}\left(\begin{array}{l}
r \\
j
\end{array}\right) \frac{1}{(j+2)((j+2) \alpha+1)} .
$$

Moreover, we have

$$
\mathcal{I}^{-1}=\alpha(\alpha+1)^{2}(\alpha+2)\left[\begin{array}{cc}
\frac{\alpha}{\alpha+2} & \frac{\theta}{\alpha+1} \\
\frac{\theta}{\alpha+1} & \frac{\theta^{2}}{\alpha^{2}}
\end{array}\right] .
$$

Hence

$$
\begin{aligned}
K_{r} & =K_{r}(\alpha):=\alpha(\alpha+1)^{2}(\alpha+2)\left(\frac{S_{r}^{2}}{\alpha(\alpha+2)}+\frac{2 S_{r} S_{r \alpha}}{\alpha+1}+S_{r \alpha}^{2}\right) \\
& =\alpha(\alpha+2)\left(S_{r}+(\alpha+1) S_{r \alpha}\right)^{2}+S_{r}^{2} .
\end{aligned}
$$

Referring to the Note following Theorem 5, we then have

Proposition 7. Goodness-of-fit tests for $F \in \operatorname{Par}_{\mathrm{T}}(\alpha, \theta)$ are given by

$$
\begin{aligned}
\widehat{D}_{n r}^{(0)}= & \frac{(2+r)^{2}(3+2 r)}{(1+r)^{2}\left(1-(2+r)^{2}(3+2 r) K_{r}\left(\widehat{\alpha}_{2 n}\right)\right)} \\
& \times \frac{1}{2 n}\left(\sum_{j=1}^{2 n}\left(1-\left(\frac{\widehat{\theta}_{2 n}}{X_{j}+\widehat{\theta}_{2 n}}\right)^{\widehat{\alpha}_{2 n}}\right)^{1+r}-\frac{2 n}{2+r}\right)^{2} \\
\stackrel{D}{\rightarrow} & \chi^{2}(1), \\
\widehat{D}_{n r}^{(1)}= & (1+r)(2+r)^{2}(3+2 r) \frac{1}{n}\left(\frac{1}{r} \sum_{j=1}^{n}\left(1-\left(\frac{\widehat{\theta}_{2 n}}{X_{j}^{*}+\widehat{\theta}_{2 n}}\right)^{\widehat{\alpha}_{2 n}}\right)^{r}\right. \\
& \left.-\frac{1}{1+r} \sum_{j=1}^{2 n}\left(1-\left(\frac{\widehat{\theta}_{2 n}}{X_{j}+\widehat{\theta}_{2 n}}\right)^{\widehat{\alpha}_{2 n}}\right)^{1+r}-\frac{2 n}{r(1+r)(2+r)}\right)^{2} \\
\stackrel{D}{\rightarrow} & \chi^{2}(1),
\end{aligned}
$$




$$
\begin{aligned}
\widehat{D}_{n r}^{(2)}= & \frac{2(1+r)(2+r)^{2}(3+2 r)}{r^{3}+r^{2}-r+1-(1-r)^{2}(1+r)(2+r)^{2}(3+2 r) K_{r}\left(\widehat{\alpha}_{2 n}\right)} \\
& \times \frac{1}{n}\left(\sum_{j=1}^{n}\left(1-\left(\frac{\widehat{\theta}_{2 n}}{X_{j}^{*}+\widehat{\theta}_{2 n}}\right)^{\widehat{\alpha}_{2 n}}\right)^{r}\right. \\
& \left.-\frac{1}{2} \sum_{j=1}^{2 n}\left(1-\left(\frac{\widehat{\theta}_{2 n}}{X_{j}+\widehat{\theta}_{2 n}}\right)^{\widehat{\alpha}_{2 n}}\right)^{1+r}-\frac{n}{2+r}\right)^{2} \\
\stackrel{D}{\rightarrow} & \chi^{2}(1), \\
\widehat{D}_{n r}^{(3)}= & \widehat{D}_{n r}^{(0)}+\widehat{D}_{n r}^{(1)} \stackrel{D}{\rightarrow} \chi^{2}(2),
\end{aligned}
$$

where

$$
\widehat{\alpha}_{2 n}=2 n / \sum_{i=1}^{2 n} \ln \left(X_{i} / \widehat{\theta}_{2 n}+1\right)
$$

and $\widehat{\theta}_{2 n}$ is obtained by numerical solution of the equation

$$
\frac{d}{d \theta} L_{2 n}\left(2 n / \sum_{i=1}^{2 n} \ln \left(x_{i} / \theta+1\right), \theta\right)=0
$$

for

$$
L_{2 n}(\alpha, \theta)=2 n \log \alpha+2 n \alpha \log \theta-(\alpha+1) \sum_{i=1}^{2 n} \log \left(x_{i}+\theta\right) .
$$

Note. Taking into account that $\partial F / \partial \theta$ can be written in the form

$$
\frac{\partial F}{\partial \theta}=-\frac{\alpha}{\theta}\left[(1-F(x))-(1-F(x))^{1+1 / \alpha}\right],
$$

we get

$$
\begin{aligned}
d_{r}(\theta) & =-\frac{\alpha}{\theta} E F^{r}(X)\left[(1-F(X))-(1-F(X))^{1+1 / \alpha}\right] \\
& =-\frac{\alpha}{\theta} \int_{0}^{1} y^{r}(1-y) d y+\frac{\alpha}{\theta} \int_{0}^{1} y^{r}(1-y)^{1+1 / \alpha} d y \\
& =\frac{\alpha}{\theta}\left[-\frac{1}{(1+r)(2+r)}+B(1+r,(2+\alpha) / \alpha)\right],
\end{aligned}
$$

and we can use in the tests the quantity

$$
\begin{aligned}
K_{r}=K_{r}(\alpha):=S_{r}^{2}+\alpha(\alpha+1)^{2}(\alpha+2) & \frac{-S_{r}}{\alpha+1}+\frac{1}{(1+r)(2+r)} \\
& -B(1+r,(2+\alpha) / \alpha))^{2} .
\end{aligned}
$$


$5^{\text {o. Let }} F \in \log (\alpha, \beta)$, i.e.

$$
\begin{aligned}
& F(x)=1 /\left[1+\exp \left(-\frac{x-\alpha}{\beta}\right)\right], \\
& f(x)=\frac{1}{\beta}\left[\exp \left(-\frac{x-\alpha}{\beta}\right)\right] /\left[\left(1+\exp \left(-\frac{x-\alpha}{\beta}\right)\right)^{2}\right]=\frac{1}{\beta} F(x)(1-F(x)) .
\end{aligned}
$$

Hence

$$
\frac{\partial F}{\partial \alpha}=-f(x), \quad \frac{\partial F}{\partial \beta}=-\frac{x-\alpha}{\beta} f(x) .
$$

Therefore

$$
\begin{aligned}
d_{r}(\alpha) & =E F^{r}(X ; \alpha, \beta) \frac{\partial F(X ; \alpha, \beta)}{\partial \alpha} \\
& =-\frac{1}{\beta} \int_{-\infty}^{\infty} F^{r}(x) F(x)(1-F(x)) f(x) d x \\
& =-\frac{1}{\beta} \int_{0}^{1} y^{1+r}(1-y) d y=-\frac{1}{\beta(r+2)(r+3)},
\end{aligned}
$$

and

$$
\begin{aligned}
d_{r}(\beta) & =E F^{r}(X ; \alpha, \beta) \frac{\partial F(X ; \alpha, \beta)}{\partial \beta} \\
& =-\frac{1}{\beta} \int_{0}^{1}\left(y^{1+r}-y^{r+2}\right) \ln \frac{y}{1-y} d y \\
& =-\frac{1}{\beta}\left[\int_{0}^{1}\left(y^{1+r}-y^{r+2}\right) \ln y d y-\int_{0}^{1}\left(y^{1+r}-y^{r+2}\right) \ln (1-y) d y\right] .
\end{aligned}
$$

Note that

$$
\int_{0}^{1} y^{r} \ln y d y=-\frac{1}{(1+r)^{2}}
$$

and

$$
\int_{0}^{1} y^{r} \ln (1-y) d y=\int_{0}^{1}(1-z)^{r} \ln z d z=-\sum_{j=0}^{\infty}(-1)^{j}\left(\begin{array}{l}
r \\
j
\end{array}\right) \frac{1}{(j+1)^{2}} .
$$

Thus

$$
\begin{aligned}
d_{r}(\beta)= & -\frac{1}{\beta}\left[-\frac{1}{(r+2)^{2}}+\frac{1}{(r+3)^{2}}\right. \\
& \left.-\left(-\sum_{j=0}^{\infty}(-1)^{j}\left(\begin{array}{c}
1+r \\
j
\end{array}\right) \frac{1}{(j+1)^{2}}+\sum_{j=0}^{\infty}(-1)^{j}\left(\begin{array}{c}
r+2 \\
j
\end{array}\right) \frac{1}{(j+1)^{2}}\right)\right]
\end{aligned}
$$




$$
\begin{aligned}
& =\frac{2 r+5}{\beta(r+2)^{2}(r+3)^{2}}-\frac{1}{\beta} \sum_{j=0}^{\infty}(-1)^{j}\left(\begin{array}{c}
1+r \\
j
\end{array}\right) \frac{1}{(j+2)^{2}} \\
& =\frac{2 r+5}{\beta(r+2)^{2}(r+3)^{2}}-\frac{1}{\beta} S_{1+r} .
\end{aligned}
$$

Moreover, we have

$$
\mathcal{I}=\frac{1}{3 \beta^{2}}\left[\begin{array}{cc}
1 & 0 \\
0 & 1+\pi^{2} / 3
\end{array}\right], \quad \mathcal{I}^{-1}=3 \beta^{2}\left[\begin{array}{cc}
1 & 0 \\
0 & 3 /\left(3+\pi^{2}\right)
\end{array}\right] .
$$

Therefore

$$
\begin{aligned}
K_{r}=\boldsymbol{d}_{r}^{\prime} \mathcal{I}^{-1} \boldsymbol{d}_{r}= & 3 \frac{1}{(r+2)^{2}(r+3)^{2}} \\
& \times\left[1+\frac{3}{3+\pi^{2}}\left(\frac{2 r+5}{(r+2)(r+3)}-(r+2)(r+3) S_{1+r}\right)^{2}\right] .
\end{aligned}
$$

Thus we have

Proposition 8. Goodness-of-fit tests for $F \in \log (\alpha, \beta)$ are given by

$$
\begin{aligned}
\widehat{D}_{n r}^{(0)}= & A_{r}^{(0)} \frac{1}{1-(1+r)^{2} A_{r}^{(0)} K_{r}} \\
& \times \frac{1}{2 n}\left(\sum_{j=1}^{2 n}\left(1+\exp \left(-\frac{X_{j}-\widehat{\alpha}_{2 n}}{\widehat{\beta}_{2 n}}\right)\right)^{-(1+r)}-\frac{2 n}{2+r}\right)^{2} \stackrel{D}{\rightarrow} \chi^{2}(1), \\
\widehat{D}_{n r}^{(1)}= & A_{r}^{(1)} \frac{1}{n}\left(\frac{1}{r} \sum_{j=1}^{n}\left(1+\exp \left(-\frac{X_{j}^{*}-\widehat{\alpha}_{2 n}}{\widehat{\beta}_{2 n}}\right)\right)^{-r}\right. \\
& \left.-\frac{1}{1+r} \sum_{j=1}^{2 n}\left(1+\exp \left(-\frac{X_{j}-\widehat{\alpha}_{2 n}}{\widehat{\beta}_{2 n}}\right)\right)^{-(1+r)}-\frac{2 n}{r(1+r)(2+r)}\right)^{2} \\
\stackrel{D}{\rightarrow} & \chi^{2}(1), \\
\widehat{D}_{n r}^{(2)}= & A_{r}^{(2)} \frac{2}{1-(1-r)^{2} A_{r}^{(2)} K_{r}} \cdot \frac{1}{n}\left(\sum_{j=1}^{n}\left(1+\exp \left(-\frac{X_{j}^{*}-\widehat{\alpha}_{2 n}}{\widehat{\beta}_{2 n}}\right)\right)^{-r}\right. \\
& \left.-\frac{1}{2} \sum_{j=1}^{2 n}\left(1+\exp \left(-\frac{X_{j}-\widehat{\alpha}_{2 n}}{\widehat{\beta}_{2 n}}\right)\right)^{-(1+r)}-\frac{n}{2+r}\right)^{2} \stackrel{D}{\rightarrow} \chi^{2}(1), \\
\widehat{D}_{n r}^{(3)}= & \widehat{D}_{n r}^{(0)}+\widehat{D}_{n r}^{(1)} \stackrel{D}{\rightarrow} \chi^{2}(2),
\end{aligned}
$$

where $A_{r}^{(0)}, A_{r}^{(1)}, A_{r}^{(2)}$ are in (3.4), and $\widehat{\alpha}_{2 n}, \widehat{\beta}_{2 n}$ are obtained by numerical solution of the equations

$$
\frac{\partial L_{2 n}}{\partial \alpha}=\frac{\partial L_{2 n}}{\partial \beta}=0
$$


for

$$
\begin{gathered}
L_{2 n}(\alpha, \beta)=-2 n \ln \beta-\frac{1}{\beta} \sum_{i=1}^{2 n}\left(x_{i}-\alpha\right)-2 \sum_{i=1}^{2 n} \ln \left(1+\exp \left(-\frac{x_{i}-\alpha}{\beta}\right)\right) . \\
\text { Numerical evaluation of } K_{r}
\end{gathered}
$$

\begin{tabular}{llllll}
\hline$r$ & 0.001 & 0.010 & 0.050 & 0.10 & 0.20 \\
$K_{r}$ & 0.08319 & 0.08196 & 0.07676 & 0.07085 & 0.06074 \\
\hline$r$ & 0.25 & 0.30 & 0.40 & 0.50 & 0.60 \\
$K_{r}$ & 0.05640 & 0.05246 & 0.04561 & 0.03990 & 0.03510 \\
\hline$r$ & 0.70 & 0.75 & 0.80 & 0.90 & 1.00 \\
$K_{r}$ & 0.03104 & 0.02924 & 0.02758 & 0.02461 & 0.02205 \\
\hline$r$ & 1.25 & 1.50 & 1.75 & 2.00 & 2.50 \\
$K_{r}$ & 0.01702 & 0.01340 & 0.01073 & 0.008714 & 0.005963 \\
\hline$r$ & 3.00 & 3.50 & 4.00 & 4.50 & 5.00 \\
$K_{r}$ & 0.004245 & 0.003120 & 0.0023536 & 0.0018147 & 0.0014255 \\
\hline
\end{tabular}

$6^{\circ}$. Let $F \in \mathrm{C}(\alpha, \beta)$, i.e.

$$
F(x)=\frac{1}{2}+\frac{1}{\pi} \arctan \left(\frac{x-\alpha}{\beta}\right), \quad f(x)=\frac{\beta}{\pi} \cdot \frac{1}{\beta^{2}+(x-\alpha)^{2}} .
$$

We see that $\frac{\partial F}{\partial \alpha}=-f(x)$ and $\frac{\partial F}{\partial \beta}=-\frac{x-\alpha}{\beta} f(x)$.

Hence

$$
\begin{aligned}
d_{r}(\alpha) & =E F^{r}(X) \frac{\partial F}{\partial \alpha}=-E\left(\frac{1}{2}+\frac{1}{\pi} \arctan \frac{X-\alpha}{\beta}\right)^{r} f(X) \\
& =-\frac{1}{\pi^{2} \beta} \int_{-\infty}^{\infty}\left(\frac{1}{2}+\frac{1}{\pi} \arctan \left(\frac{x-\alpha}{\beta}\right)\right)^{r} \frac{1}{\left(1+\left(\frac{x-\alpha}{\beta}\right)^{2}\right)^{2}} d x \\
& =-\frac{1}{\pi \beta} \int_{0}^{1} y^{r} \cos ^{2} \pi\left(y-\frac{1}{2}\right) d y=-\frac{1}{\pi \beta} \int_{0}^{1} y^{r} \frac{1+\cos (2 \pi y-\pi)}{2} d y \\
& =-\frac{1}{2 \pi \beta} \int_{0}^{1}(1-\cos 2 \pi y) d y=\frac{1}{2 \pi \beta} \sum_{j=1}^{\infty}(-1)^{j} \frac{(2 \pi)^{2 j}}{(2 j) !} \int_{0}^{1} y^{r+2 j} d y \\
& =\frac{1}{2 \pi \beta} \sum_{j=1}^{\infty}(-1)^{j} \frac{(2 \pi)^{2 j}}{(2 j) !(2 j+1+r)}
\end{aligned}
$$

and

$$
\begin{aligned}
d_{r}(\beta) & =E F^{r}(X) \frac{\partial F}{\partial \beta}=-E\left(\frac{1}{2}+\frac{1}{\pi} \arctan \left(\frac{X-\alpha}{\beta}\right)\right)^{r} \frac{X-\alpha}{\beta} f(X) \\
& =-\frac{1}{\pi^{2} \beta^{2}} \int_{-\infty}^{\infty}\left(\frac{1}{2}+\frac{1}{\pi} \arctan \frac{x-\alpha}{\beta}\right)^{r} \frac{x-\alpha}{\beta} \frac{d x}{\left(1+\left(\frac{x-\alpha}{\beta}\right)^{2}\right)^{2}}
\end{aligned}
$$




$$
\begin{aligned}
& =-\frac{1}{\pi \beta} \int_{0}^{1} y^{r} \tan \pi\left(y-\frac{1}{2}\right) \cos ^{2} \pi\left(y-\frac{1}{2}\right) d y \\
& =-\frac{1}{2 \pi \beta} \int_{0}^{1} y^{2} \sin 2 \pi\left(y-\frac{1}{2}\right) d y=\frac{1}{2 \pi \beta} \int_{0}^{1} y^{2} \sin 2 \pi y d y \\
& =\frac{1}{2 \pi \beta} \sum_{j=0}^{\infty}(-1)^{j} \frac{(2 \pi)^{2 j+1}}{(2 j+1) !} \int_{0}^{1} y^{r+2 j+1} d y \\
& =\frac{1}{2 \pi \beta} \sum_{j=0}^{\infty}(-1)^{j} \frac{(2 \pi)^{2 j+1}}{(2 j+1) !(2 j+2+r)} .
\end{aligned}
$$

Hence

$$
\boldsymbol{d}_{r}=\frac{1}{2 \pi \beta}\left[\begin{array}{c}
\sum_{j=1}^{\infty}(-1)^{j} \frac{(2 \pi)^{2 j}}{(2 j) !(2 j+1+r)} \\
-\sum_{j=1}^{\infty}(-1)^{j} \frac{(2 \pi)^{2 j-1}}{(2 j-1) !(2 j+r)}
\end{array}\right]
$$

and taking into account that

we have

$$
\mathcal{I}^{-1}=2 \beta^{2}\left[\begin{array}{ll}
1 & 0 \\
0 & 1
\end{array}\right]
$$

$$
\begin{aligned}
K_{r}= & \frac{1}{2 \pi^{2}}\left[\left(\sum_{j=1}^{\infty}(-1)^{j} \frac{(2 \pi)^{2 j}}{(2 j+1+r)(2 j) !}\right)^{2}\right. \\
& \left.+\left(\sum_{j=1}^{\infty}(-1)^{j} \frac{(2 \pi)^{2 j-1}}{(2 j+r)(2 j-1) !}\right)^{2}\right] .
\end{aligned}
$$

Thus we get

Proposition 9. Goodness-of-fit tests for $F \in \mathrm{C}(\alpha, \beta)$ are given by

$$
\begin{aligned}
\widehat{D}_{n r}^{(0)}= & A_{r}^{(0)} \frac{1}{1-(1+r)^{2} A_{r}^{(0)} K_{r}} \cdot \frac{1}{2 n} \\
& \times\left(\sum_{j=1}^{2 n}\left(\frac{1}{2}+\frac{1}{\pi} \arctan \left(\frac{X_{j}-\widehat{\alpha}_{2 n}}{\widehat{\beta}_{2 n}}\right)^{1+r}-\frac{2 n}{2+r}\right)\right)^{2} \stackrel{D}{\rightarrow} \chi^{2}(1), \\
\widehat{D}_{n r}^{(1)}= & A_{r}^{(1)} \frac{1}{n}\left(\frac{1}{r} \sum_{j=1}^{n}\left(\frac{1}{2}+\frac{1}{\pi} \arctan \left(\frac{X_{j}^{*}-\widehat{\alpha}_{2 n}}{\widehat{\beta}_{2 n}}\right)\right)^{r}\right) \\
& \left.-\frac{1}{1+r} \sum_{j=1}^{2 n}\left(\frac{1}{2}+\frac{1}{\pi} \arctan \left(\frac{X_{j}-\widehat{\alpha}_{2 n}}{\widehat{\beta}_{2 n}}\right)\right)^{1+r}-\frac{2 n}{r(1+r)(r+2)}\right)^{2} \\
\stackrel{D}{\rightarrow} & \chi^{2}(1),
\end{aligned}
$$




$$
\begin{aligned}
\widehat{D}_{n r}^{(2)}= & A_{r}^{(2)} \frac{2}{1-(1-r)^{2} A_{r}^{(2)} K_{r}} \cdot \frac{1}{n}\left(\sum_{j=1}^{n}\left(\frac{1}{2}+\frac{1}{\pi} \arctan \left(\frac{X_{j}^{*}-\widehat{\alpha}_{2 n}}{\widehat{\beta}_{2 n}}\right)\right)^{r}\right. \\
& \left.-\frac{1}{2} \sum_{j=1}^{2 n}\left(\frac{1}{2}+\frac{1}{\pi} \arctan \left(\frac{X_{j}-\widehat{\alpha}_{2 n}}{\widehat{\beta}_{2 n}}\right)\right)^{1+r}-\frac{n}{r+2}\right)^{2} \stackrel{D}{\rightarrow} \chi^{2}(1), \\
\widehat{D}_{n r}^{(3)}= & \widehat{D}_{n r}^{(0)}+\widehat{D}_{n r}^{(1)} \stackrel{D}{\rightarrow} \chi^{2}(2),
\end{aligned}
$$

where $A_{r}^{(0)}, A_{r}^{(1)}, A_{r}^{(2)}$ are in (3.4), and $\widehat{\alpha}_{2 n}$ and $\widehat{\beta}_{2 n}$ are obtained by solving numerically the equations

$$
\frac{\partial L_{2 n}}{\partial \alpha}=\frac{\partial L_{2 n}}{\partial \beta}=0
$$

for

$$
L_{2 n}(\alpha, \beta)=\sum_{i=1}^{2 n}\left(-\ln \pi+\ln \beta-\ln \left[\beta^{2}+\left(x_{i}-\alpha\right)^{2}\right]\right) .
$$

Numerical evaluation of $K_{r}$

\begin{tabular}{llllll}
\hline$r$ & 0.001 & 0.010 & 0.050 & 0.10 & 0.20 \\
$K_{r}$ & 0.05058 & 0.04988 & 0.04693 & 0.04354 & 0.03765 \\
\hline$r$ & 0.25 & 0.30 & 0.40 & 0.50 & 0.60 \\
$K_{r}$ & 0.03508 & 0.03274 & 0.02862 & 0.02513 & 0.02217 \\
\hline$r$ & 0.70 & 0.75 & 0.80 & 0.90 & 1.00 \\
$K_{r}$ & 0.01964 & 0.01851 & 0.01746 & 0.01558 & 0.01395 \\
\hline$r$ & 1.25 & 1.50 & 1.75 & 2.00 & 2.50 \\
$K_{r}$ & 0.010718 & 0.008374 & 0.006639 & 0.005331 & 0.003553 \\
\hline$r$ & 3.00 & 3.50 & 4.00 & 4.50 & 5.00 \\
$K_{r}$ & 0.002457 & 0.001751 & 0.0012810 & 0.0009577 & 0.0007298 \\
\hline
\end{tabular}

$7^{\circ}$. Let $F \in \mathrm{N}(\mu, \sigma)$, i.e.

$$
F(x)=\frac{1}{\sqrt{2 \pi} \sigma} \int_{-\infty}^{x} e^{-\frac{(t-\mu)^{2}}{2 \sigma^{2}}} d t, \quad f(x)=\frac{1}{\sigma \sqrt{2 \pi}} e^{-\frac{(x-\mu)^{2}}{2 \sigma^{2}}} .
$$

Note that

$$
\frac{\partial F}{\partial \mu}=-f(x), \quad \frac{\partial F}{\partial \sigma^{2}}=-\frac{x-\mu}{2 \sigma^{2}} f(x) .
$$

Now we use the probability function

$$
\operatorname{erf} z=\frac{2}{\sqrt{\pi}} \int_{0}^{z} e^{-y^{2}} d y, \quad z \in \mathbb{R} .
$$

Then

$$
F(x)=\frac{1}{2}+\frac{1}{2} \operatorname{erf}\left(\frac{x-\mu}{\sigma \sqrt{2}}\right)
$$


Hence

$$
\begin{aligned}
d_{r}(\mu) & =E F^{r}(X) \frac{\partial F}{\partial \mu}=-E F^{r}(X) f(X) \\
& =-\int_{-\infty}^{\infty}\left(\frac{1}{2}+\frac{1}{2} \operatorname{erf}\left(\frac{x-\mu}{\sigma \sqrt{2}}\right)\right)^{r} \frac{1}{2 \pi \sigma^{2}} e^{-\frac{(x-\mu)^{2}}{\sigma^{2}}} d x \\
& =-\frac{1}{2^{r} \sqrt{2} \pi \sigma} \int_{-\infty}^{\infty}(1+\operatorname{erf} z)^{r} e^{-2 z^{2}} d z \\
& =-\frac{1}{2^{r} \sqrt{2} \pi \sigma} \sum_{j=0}^{\infty}\left(\begin{array}{c}
r \\
j
\end{array}\right) \int_{-\infty}^{\infty}(\operatorname{erf} z)^{j} e^{-2 z^{2}} d z \\
& =-\frac{1}{2^{1+r} \sqrt{\pi} \sigma}-\frac{1}{2^{r} \sqrt{2} \pi \sigma} \sum_{j=1}^{\infty}\left(\begin{array}{c}
r \\
j
\end{array}\right) \int_{-\infty}^{\infty}(\operatorname{erf} z)^{j} e^{-2 z^{2}} d z \\
& =-\frac{1}{2^{1+r} \sqrt{\pi} \sigma}-\frac{1}{2^{r-1} \sqrt{2} \pi \sigma} \sum_{j=1}^{\infty}\left(\begin{array}{c}
r \\
2 j
\end{array}\right) \int_{0}^{\infty}(\operatorname{erf} z)^{2 j} e^{-2 z^{2}} d z
\end{aligned}
$$

as

$$
\int_{-\infty}^{\infty}(\operatorname{erf} z)^{2 j+1} e^{-2 z^{2}} d z=0, \quad j=0,1,2, \ldots
$$

and

$$
\begin{aligned}
d_{r}\left(\sigma^{2}\right)= & E F^{r}(X) \frac{\partial F}{\partial \sigma^{2}}=-E F^{r}(X) \frac{X-\mu}{2 \sigma^{2}} f(X) \\
= & -\int_{-\infty}^{\infty}\left(\frac{1}{2}+\frac{1}{2} \operatorname{erf}\left(\frac{x-\mu}{\sigma \sqrt{2}}\right)\right)^{r} \frac{x-\mu}{2 \sigma^{2}} \cdot \frac{1}{2 \pi \sigma^{2}} e^{-\frac{(x-\mu)^{2}}{\sigma^{2}}} d x \\
= & -\frac{1}{2^{1+r} \pi \sigma^{2}} \int_{-\infty}^{\infty}(1+\operatorname{erf} z)^{r} z e^{-2 z^{2}} d z \\
= & -\frac{1}{2^{1+r} \pi \sigma^{2}} \sum_{j=0}^{\infty}\left(\begin{array}{l}
r \\
j
\end{array}\right) \int_{-\infty}^{\infty}(\operatorname{erf} z)^{j} z e^{-2 z^{2}} d z \\
= & -\frac{r}{2^{1+r} \pi \sigma^{2}} \int_{-\infty}^{\infty}(\operatorname{erf} z) z e^{-2 z^{2}} d z \\
& -\frac{1}{2^{1+r} \pi \sigma^{2}} \sum_{j=3}^{\infty}\left(\begin{array}{l}
r \\
j
\end{array}\right) \int_{-\infty}^{\infty}(\operatorname{erf} z)^{j} z e^{-2 z^{2}} d z \\
= & -\frac{r}{2^{2+r} \sqrt{3} \pi \sigma^{2}}-\frac{1}{2^{r} \pi \sigma^{2}} \sum_{j=1}^{\infty}\left(\begin{array}{c}
r \\
2 j+1
\end{array}\right) \int_{0}^{\infty}(\mathrm{erf} z)^{2 j+1} z e^{-2 z^{2}} d z
\end{aligned}
$$


as $z \operatorname{erf} z$ is an even function. Hence

$$
\boldsymbol{d}_{r}=\frac{1}{2^{1+r} \sqrt{\pi} \sigma}\left[\begin{array}{c}
-1-2 \sqrt{2 / \pi} W_{1 r} \\
-\frac{r}{2 \sqrt{3 \pi} \sigma}-\frac{2}{\sqrt{\pi} \sigma} W_{2 r}
\end{array}\right]
$$

where

$$
\begin{aligned}
& W_{1 r}=\sum_{j=1}^{\infty}\left(\begin{array}{c}
r \\
2 j
\end{array}\right) \int_{0}^{\infty}(\operatorname{erf} z)^{2 j} e^{-2 z^{2}} d z \\
& W_{2 r}=\sum_{j=1}^{\infty}\left(\begin{array}{c}
r \\
2 j+1
\end{array}\right) \int_{0}^{\infty}(\operatorname{erf} z)^{2 j+1} z e^{-2 z^{2}} d z
\end{aligned}
$$

Using the fact that

$$
\mathcal{I}^{-1}=\sigma^{2}\left[\begin{array}{cc}
1 & 0 \\
0 & 2 \sigma^{2}
\end{array}\right]
$$

we get

$$
K_{r}=\frac{1}{2^{2(1+r)} \pi}\left[\left(1+2 \sqrt{\frac{2}{\pi}} W_{1 r}\right)^{2}+\frac{2}{\pi}\left(\frac{r}{2 \sqrt{3}}+2 W_{2 r}\right)^{2}\right] .
$$

Proposition 10. Goodness-of-fit tests for $\mathrm{N}(\mu, \sigma)$ are given by

$$
\begin{aligned}
& \widehat{D}_{n r}^{(0)}=A_{r}^{(0)} \frac{1}{1-(1+r)^{2} A_{r}^{(0)} K_{r}} \\
& \times \frac{1}{2 n}\left(\sum_{j=1}^{2 n} \Phi^{1+r}\left(\frac{X_{j}-\widehat{\mu}_{2 n}}{\widehat{\sigma}_{2 n}}\right)-\frac{2 n}{2+r}\right)^{2} \\
& \stackrel{D}{\rightarrow} \chi^{2}(1), \\
& \widehat{D}_{n r}^{(1)}=A_{r}^{(1)} \frac{1}{n}\left(\frac{1}{r} \sum_{j=1}^{n} \Phi^{r}\left(\frac{X_{j}^{*}-\widehat{\mu}_{2 n}}{\widehat{\sigma}_{2 n}}\right)\right. \\
& \left.-\frac{1}{1+r} \sum_{j=1}^{2 n} \Phi^{1+r}\left(\frac{X_{j}-\widehat{\mu}_{2 n}}{\widehat{\sigma}_{2 n}}\right)-\frac{2 n}{r(1+r)(2+r)}\right)^{2} \\
& \stackrel{D}{\rightarrow} \chi^{2}(1), \\
& \widehat{D}_{n r}^{(2)}=A_{r}^{(2)} \frac{2}{1-(1-r)^{2} A_{r}^{(2)} K_{r}} \cdot \frac{1}{n}\left(\sum_{j=1}^{n} \Phi^{r}\left(\frac{X_{j}^{*}-\widehat{\mu}_{2 n}}{\widehat{\sigma}_{2 n}}\right)\right. \\
& \left.-\frac{1}{2} \sum_{j=1}^{2 n} \Phi^{1+r}\left(\frac{X_{j}-\widehat{\mu}_{2 n}}{\widehat{\sigma}_{2 n}}\right)-\frac{n}{2+r}\right)^{2} \stackrel{D}{\rightarrow} \chi^{2}(1), \\
& \widehat{D}_{n r}^{(3)}=\widehat{D}_{n r}^{(0)}+\widehat{D}_{n r}^{(1)} \stackrel{D}{\rightarrow} \chi^{2}(2),
\end{aligned}
$$


where $A_{r}^{(0)}, A_{r}^{(1)}, A_{r}^{(2)}$ are in (3.4), and

$$
\begin{gathered}
\Phi(x)=\frac{1}{\sqrt{2 \pi}} \int_{-\infty}^{x} e^{-t^{2} / 2} d t \\
\widehat{\mu}_{2 n}=\bar{X}_{2 n}, \quad \widehat{\sigma}_{2 n}^{2}=\frac{1}{2 n} \sum_{j=1}^{2 n}\left(X_{j}-\bar{X}_{2 n}\right)^{2} .
\end{gathered}
$$

Numerical evaluation of $K_{r}$

\begin{tabular}{llllll}
\hline$r$ & 0.001 & 0.010 & 0.050 & 0.10 & 0.20 \\
$K_{r}$ & 0.07944 & 0.07824 & 0.07319 & 0.0674736 & 0.0577260 \\
\hline$r$ & 0.25 & 0.30 & 0.40 & 0.50 & 0.60 \\
$K_{r}$ & 0.0535579 & 0.0497855 & 0.0432499 & 0.0378199 & 0.0332698 \\
$r$ & 0.70 & 0.75 & 0.80 & 0.90 & 1.00 \\
$K_{r}$ & 0.0294272 & 0.0277282 & 0.0261587 & 0.0233603 & 0.0209498 \\
$r r$ & 1.25 & 1.50 & 1.75 & 2.00 & 2.50 \\
$K_{r}$ & 0.0162222 & 0.0128211 & 0.0103097 & 0.0084138 & 0.0058144 \\
\hline$r$ & 3.00 & 3.50 & 4.00 & 4.50 & 5.00 \\
$K_{r}$ & 0.0041813 & 0.0031037 & 0.0023642 & 0.0018401 & 0.0014586 \\
\hline
\end{tabular}

$8^{\circ}$. Let $F \in \operatorname{EV}(\alpha, \beta)$, i.e.

$$
\begin{aligned}
& F(x)=\exp \left(-\exp \left(-\frac{x-\alpha}{\beta}\right)\right), \quad \alpha \in \mathbb{R}, \beta>0, \\
& f(x)=\frac{1}{\beta} \exp \left(-e^{-\frac{x-\alpha}{\beta}}\right) e^{-\frac{x-\alpha}{\beta}}=\frac{1}{\beta} F(x) \ln \frac{1}{F(x)} .
\end{aligned}
$$

Then

$$
\begin{aligned}
& \frac{\partial F}{\partial \alpha}=\frac{1}{\beta} F(x) \ln F(x), \\
& \frac{\partial F}{\partial \beta}=\frac{1}{\beta} F(x)(-\ln F(x)) \ln (-\ln F(x)) .
\end{aligned}
$$

Here we use the following integrals:

$$
\int_{0}^{1} x^{p-1}(-\ln x)^{q-1} \ln (-\ln x) d x=\frac{\Gamma(q)}{p^{q}}(\psi(q)-\ln p), \quad p>0, q>0,
$$

where

$$
\psi(q)=\frac{\Gamma^{\prime}(q)}{\Gamma(q)}, \quad \psi(q+1)=\frac{1}{q}+\psi(q), \quad \psi(1)=-\gamma
$$

whence

$$
\int_{0}^{1} y^{1+r}(-\ln y) \ln (-\ln y) d y=\frac{1}{(2+r)^{2}}(1-\gamma-\ln (2+r)) .
$$


Then

$$
\begin{aligned}
d_{r}(\alpha) & =E F^{r}(X) \frac{\partial F}{\partial \alpha}=\frac{1}{\beta} \int_{-\infty}^{\infty} F^{1+r}(x) \ln F(x) f(x) d x \\
& =\frac{1}{\beta} \int_{0}^{1} y^{1+r} \ln y d y=-\frac{1}{\beta} \frac{1}{(2+r)^{2}}
\end{aligned}
$$

and similarly

$$
\begin{aligned}
d_{r}(\beta) & =E F^{r}(X) \frac{\partial F}{\partial \beta}=\frac{1}{\beta} \int_{-\infty}^{\infty} F^{1+r}(x)(-\ln F(x)) \ln (-\ln F(x)) f(x) d x \\
& =\frac{1}{\beta} \int_{0}^{1} y^{1+r}(-\ln y) \ln (-\ln y) d y=\frac{1}{\beta} \cdot \frac{1}{(2+r)^{2}}(1-\gamma-\ln (2+r)) .
\end{aligned}
$$

Hence

$$
\boldsymbol{d}_{r}=\frac{1}{\beta(2+r)^{2}}\left[\begin{array}{c}
-1 \\
1-\gamma-\ln (2+r)
\end{array}\right]
$$

Using the fact that

$$
\mathcal{I}^{-1}=\frac{6 \beta^{2}}{\pi^{2}}\left[\begin{array}{cc}
(1-\gamma)^{2}+\pi^{2} / 6 & 1-\gamma \\
1-\gamma & 1
\end{array}\right]
$$

we get

$$
K_{r}=\frac{1}{(2+r)^{4}}\left[1+\frac{6}{\pi^{2}} \ln ^{2}(2+r)\right]
$$

Thus we have

Proposition 11. Goodness-of-fit tests for $F \in \operatorname{EV}(\alpha, \beta)$ are given by

$$
\begin{aligned}
\widehat{D}_{n r}^{(0)}= & A_{r}^{(0)} \frac{1}{1-(1+r)^{2} A_{r}^{(0)} K_{r}} \\
& \times \frac{1}{2 n}\left(\sum_{j=1}^{2 n} \exp \left((1+r) \exp \left(-\frac{X_{j}-\widehat{\alpha}_{2 n}}{\widehat{\beta}_{2 n}}\right)\right)-\frac{2 n}{2+r}\right)^{2} \\
\stackrel{D}{\rightarrow} & \chi^{2}(1) \\
\widehat{D}_{n r}^{(1)}= & A_{r}^{(1)} \frac{1}{n}\left(\frac{1}{r} \sum_{j=1}^{n} \exp \left(-r \exp \left(-\frac{X_{j}^{*}-\widehat{\alpha}_{2 n}}{\widehat{\beta}_{2 n}}\right)\right)\right. \\
& \left.-\frac{1}{1+r} \sum_{j=1}^{2 n} \exp \left(-(1+r) \exp \left(-\frac{X_{j}^{*}-\widehat{\alpha}_{2 n}}{\widehat{\beta}_{2 n}}\right)\right)-\frac{2 n}{r(1+r)(2+r)}\right)^{2} \\
\stackrel{D}{\rightarrow} & \chi^{2}(1),
\end{aligned}
$$




$$
\begin{aligned}
\widehat{D}_{n r}^{(2)}= & A_{r}^{(2)} \frac{2}{1-(1-r)^{2} A_{r}^{(2)} K_{r}} \cdot \frac{1}{n}\left(\sum_{j=1}^{n} \exp \left(-r \exp \left(-\frac{X_{j}^{*}-\widehat{\alpha}_{2 n}}{\widehat{\beta}_{2 n}}\right)\right)\right. \\
& \left.-\frac{1}{2} \sum_{j=1}^{2 n} \exp \left(-(1+r) \exp \left(-\frac{X_{j}^{*}-\widehat{\alpha}_{2 n}}{\widehat{\beta}_{2 n}}\right)\right)-\frac{n}{2+r}\right)^{2} \stackrel{D}{\rightarrow} \chi^{2}(1), \\
\widehat{D}_{n r}^{(3)}= & \widehat{D}_{n r}^{(0)}+\widehat{D}_{n r}^{(1)} \stackrel{D}{\rightarrow} \chi^{2}(2),
\end{aligned}
$$

where $A_{r}^{(0)}, A_{r}^{(1)}, A_{r}^{(2)}$ are in (3.4), and $\widehat{\alpha}_{2 n}$ and $\widehat{\beta}_{2 n}$ are obtained by solving numerically the equations

$$
\frac{\partial L_{2 n}}{\partial \alpha}=\frac{\partial L_{2 n}}{\partial \beta}=0
$$

for

$$
L_{2 n}(\alpha, \beta)=-2 n \ln \beta-\sum_{i=1}^{2 n} \exp \left(-\frac{x_{i}-\alpha}{\beta}\right)-\frac{2 n}{\beta}\left(\bar{X}_{2 n}-\alpha\right)
$$

Numerical evaluation of $K_{r}$

\begin{tabular}{llllll}
\hline$r$ & 0.001 & 0.010 & 0.050 & 0.10 & 0.20 \\
$K_{r}$ & 0.08062 & 0.07942 & 0.07436 & 0.06863 & 0.05882 \\
\hline$r$ & 0.25 & 0.30 & 0.40 & 0.50 & 0.60 \\
$K_{r}$ & 0.05462 & 0.05081 & 0.04418 & 0.03867 & 0.03403 \\
\hline$r$ & 0.70 & 0.75 & 0.80 & 0.90 & 1.00 \\
$K_{r}$ & 0.03010 & 0.02836 & 0.02675 & 0.02388 & 0.02140 \\
\hline$r$ & 1.25 & 1.50 & 1.75 & 2.00 & 2.50 \\
$K_{r}$ & 0.016533 & 0.013022 & 0.010427 & 0.008470 & 0.005792 \\
\hline$r$ & 3.00 & 3.50 & 4.00 & 4.50 & 5.00 \\
$K_{r}$ & 0.004120 & 0.003024 & 0.0022775 & 0.0017534 & 0.0013752 \\
\hline
\end{tabular}

Acknowledgements. The authors are grateful to the referee for a number of useful suggestions and comments.

\section{References}

[1] B. C. Arnold, Pareto Distributions, Internat. Co-oper. Publ. House, Burtonsville, MD, 1983.

[2] M. Csörgö, V. Seshadri and M. Yalovsky, Applications of characterizations in the area of goodness of fit, in: C. P. Patil et al. (eds.), Statistical Distributions in Scientific Work, Vol. 2, Reidel, Dordrecht, 1975, 79-90.

[3] - - - - Some exact tests for normality in the presence of unknown parameters, J. Roy. Statist. Soc. Ser. B 35 (1973), 507-522.

[4] R. B. D'Agostino and M. A. Stephens, Goodness-of-fit Techniques, Dekker, New York, 1986.

[5] N. Ebrahimi and M. Habibullah, Testing exponentiality based on Kullback-Leibler information, J. Roy. Statist. Soc. Ser. B 54 (1992), 739-748. 
[6] Z. Grudzień and D. Szynal, Characterization of continuous distributions in terms of moments of extremal statistics, J. Math. Sci. 81 (1996), 2912-2936.

[7] —, - Characterizations of continuous distributions via moments of order statistics and record values, Brandenburg. Techn. Univer. Cottbus, Fakultät für Math., Naturwiss. und Informatik, Reihe Math., M-05/1997 (1997).

[8] T. Hashimoto and S. Shirahata, A goodness of fit test based on the characterization of uniform distribution, J. Japan Statist. Soc. 23 (1993), 123-130.

[9] T. Inglot and T. Ledwina, Asymptotic optimality of data-driven Neyman's tests for uniformity, Ann. Statist. 24 (1996), 1982-2019.

[10] N. L. Johnson and S. Kotz, Continuous Univariate Distributions-1, Houghton Mifflin, Boston, 1970, p. 256.

[11] S. A. Klugman, H. H. Panjer and G. E. Willmot, Loss Models. From Data to Decisions, Wiley, 1998.

[12] G. D. Lin, Characterizations of continuous distributions via expected values of two functions of order statistics, Sankhyā Ser. A 52 (1990), 84-90.

[13] K. McDonald and S. K. Katti, Test for normality using a characterization, C. P. Patil et al. (eds.), Statistical Distributions in Scientific Work, Vol. 2, Reidel, Dordrecht, 1975, 91-104.

[14] K. Morris and D. Szynal, A goodness-of-fit test for the uniform distribution based on a characterization, J. Math. Sci. 106 (2001), 2719-2724.

[15] —, Goodness-of-fit tests based on characterizations of continuous distributions, Appl. Math. 27 (2000), 475-488.

[16] - Tests for uniformity and exponentiality using characterization, submitted.

[17] - Goodness-of-fit tests using characterizations of continuous distributions, Appl. Math. 28 (2001), 151-168.

[18] D. A. Pierce, The asymptotic effect of substituting estimators for parameters in certain types of statistics, Ann. Statist. 10 (1982), 475-478.

[19] R. H. Randles, On the asymptotic normality of statistics with estimated parameters, ibid. 10 (1982), 462-474.

[20] J. C. W. Rayner and D. J. Best, Smooth tests for goodness of fit: an overview, Int. Statist. Rev. 58 (1990), 9-17.

[21] —, - Smooth Tests of Goodness of Fit, Oxford Univ. Press, Oxford, 1989.

[22] I. M. Ryzhik and I. S. Gradshtel̆n, Tables of Integrals, Sums, Series and Products, PWN, Warszawa, 1964 (in Polish).

[23] Y. H. Too and G. D. Lin, Characterizations of uniform and exponential distributions, Statist. Probab. Lett. 7 (1989), 357-359.

Department of Applied Mathematics

University of Adelaide

North Tce, Adelaide

South Australia, 5001

E-mail: kmorris@stats.adelaide.edu.au
Institute of Mathematics

Maria Curie-Skłodowska University pl. M. Curie-Skłodowskiej 1 20-031 Lublin, Poland

E-mail: szynal@golem.umcs.lublin.pl

Received on 11.10.2001;

revised version on 7.5.2002 